PAPER: DISORDERED SYSTEMS, CLASSICAL AND QUANTUM

\section{Random-link matching problems on random regular graphs}

To cite this article: Giorgio Parisi et al J. Stat. Mech. (2020) 033301

View the article online for updates and enhancements.
You may also like

$$
\begin{aligned}
& \text { - Bifurcations in synergistic epidemics on } \\
& \frac{\text { random reqular graphs }}{\text { S N Taraskin and F J Pérez-Reche }} \\
& \text { - Analytical results for the distribution of first } \\
& \frac{\frac{\text { return times of random walks on random }}{\text { reqular graphs }}}{\text { Ido Tishby, Ofer Biham and Eytan Katzav }} \\
& \text { - Moments of the inverse participation ratio } \\
& \frac{\text { for the Laplacian on finite regular graphs }}{\text { Timothy B P Clark and Adrian Del Maestro }}
\end{aligned}
$$

\section{Recent citations}

Criticality and conformality in the random

S. Caracciolo et al

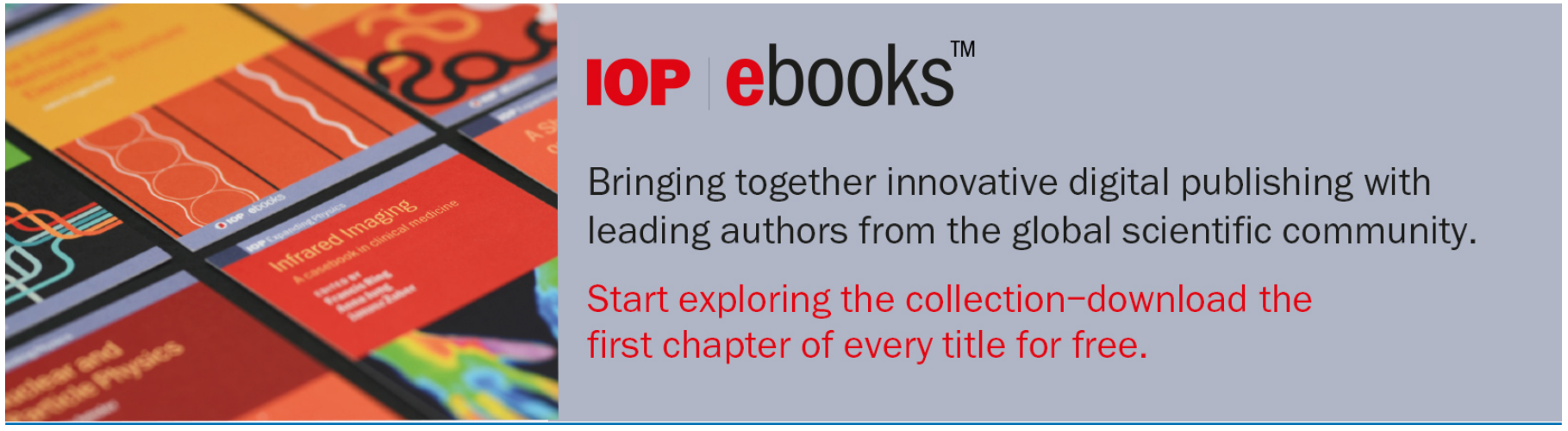

This content was downloaded from IP address 5.170.108.224 on 29/11/2021 at 09:00 


\title{
Random-link matching problems on random regular graphs
}

\section{Giorgio Parisi ${ }^{1,2}$, Gianmarco Perrupato ${ }^{1}$ and Gabriele Sicuro ${ }^{1}$}

${ }^{1}$ Dipartimento di Fisica, Sapienza Università di Roma, P.le A. Moro 2, I-00185, Rome, Italy

2 INFN_Sezione di Roma1, CNR-IPCF UOS Roma Kerberos, P.le A. Moro 2, I-00185, Rome, Italy

E-mail: gianmarco.perrupato@uniroma1.it

Received 9 October 2019

Accepted for publication 9 January 2020

Published 11 March 2020

Online at stacks.iop.org/JSTAT/2020/033301

https://doi.org/10.1088/1742-5468/ab7127

\begin{abstract}
We study the random-link matching problem on random regular graphs, along with two relaxed versions of the problem, namely the fractional matching and the so-called 'loopy' fractional matching. We estimate the asymptotic average optimal cost using the cavity method. Moreover, we study the finite-size corrections due to rare topological structures appearing in the graph at large sizes. We estimate these contributions using the cavity approach, and we compare our results with the output of numerical simulations. The analysis also clarifies the meaning of the finite-size contributions appearing in the fully connected version of the problem, which have already been analyzed in the literature.
\end{abstract}

Keywords: cavity and replica methods, exact results, random graphs, networks 


\section{Contents}

1. Introduction 2

2. The random-link matching problem 4

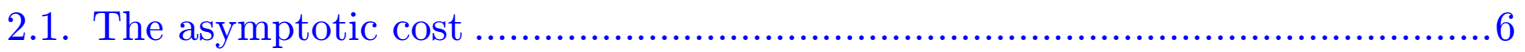

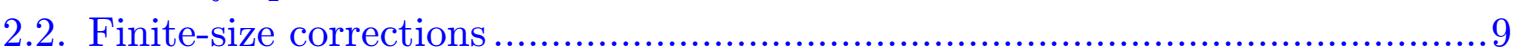

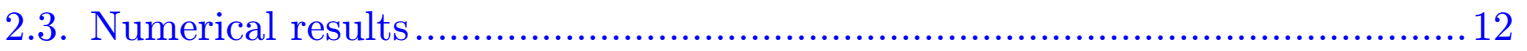

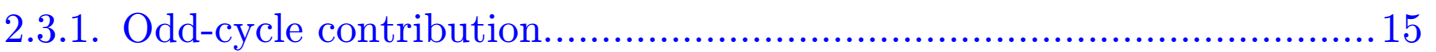

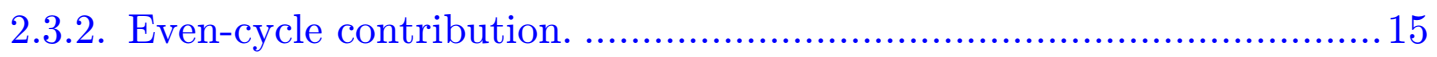

2.3.3. Wrapping up...................................................................... 16

2.4. A note on the assignment problem ............................................... 17

3. The fractional matching problem $\quad 18$

4. The 'loopy' FMP 19

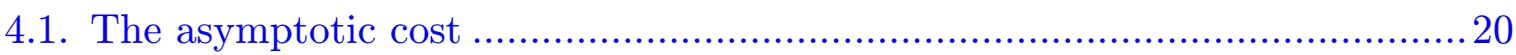

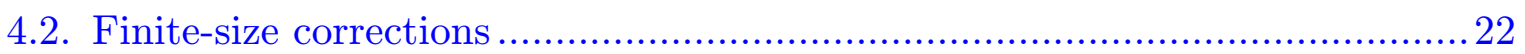

5. The fully connected limit $\quad 22$

6. Conclusions 24

Acknowledgments ............................................................. 25

Appendix. Finite- $z$ corrections .............................................. 25

A.1. Loopy fractional random matching......................................................2 28

$\begin{array}{ll}\text { References } & \mathbf{2 9}\end{array}$

\section{Introduction}

The matching problem is a classical combinatorial optimization problem that has been widely investigated by mathematicians and computer scientists [1]. The problem also has interesting relations with fundamental problems in physics: the correspondence between dimer covering problems and the Ising model on lattices, for example, was first highlighted in the sixties [2]. In the matching problem, the target is to find a dimer covering on a given graph, in such a way that no node remains unpaired. If the graph is weighted, then it is typically interesting to find the dimer covering of minimum or maximum 'cost', defined as the sum of the weights of the selected edges. From the computational point of view, the matching problem is an 'easy problem' belonging to the $\mathrm{P}$ complexity class, and polynomial algorithms are available for its solution [3-6]. It has been soon realized that solving a (weighted) matching problem is equivalent to finding the ground state of a system having many possible configurations (possible matchings) of different 'energy'. This physical point of view has been widely exploited and applied to combinatorial optimization problems in general: a Hamiltonian is associated with 
the cost function that has to be minimized, and the ground state (corresponding to the optimal solution) is obtained considering the zero-temperature limit [7].

When the effectiveness of statistical physics ideas in the study of optimization problems became evident, the matching problem started playing the role of 'toy model', due to its simplicity and relevance. In particular, the theory of disordered systems seems to be especially suitable for the study of the typical properties of optimization problems in the presence of randomness [8-11]. Exploration of this topic, which also inspired powerful numerical techniques for the algorithmic solution of these problems, started in the eighties, with the seminal works of Orland [12] and Mézard and Parisi [13], who tackled, as a prototype problem, the random-link matching problem (MP) on a complete graph. They proved that the MP can be fully investigated using the replica method and the cavity method in the limit of a large number of vertices [14]. Exact results have been obtained related to the leading-order cost and finite-size corrections [15-17], the fluctuation of the average optimal cost (AOC) [18], and its embedding in Euclidean space [19-21].

Despite its popularity in the fully connected version, very few results are available on the MP on sparse topologies. Zhou and Ou-Yang [22] and subsequently Zdeborová and Mézard [23] studied, using the cavity method, the number of maximum and perfect (unweighted) matchings on sparse graphs. Their results were later rigorously proved by Bordenave, Lelarge and Salez [24]. As far as we know, a study of the random weighted matching problem on the Bethe lattice is missing in the literature.

In this paper, we investigate exactly this formulation of the matching problem using the cavity method, both at the leading order and at the level of finite-size corrections. This analysis will be, first of all, of methodological interest: indeed, we will check, for the first time to our knowledge, the effectiveness of the cavity method in the evaluation of the contribution to finite-size corrections of rare topological structures in a combinatorial optimization problem defined on a sparse graph. Such an approach, introduced in the study of spin glasses in [25-27], allows us to go beyond the leading order using results (like the cavity field distribution) obtained at the leading level. More recently, Coja-Oghlan and coworkers [28] have rigorously grounded the cavity method results for a large class of models, characterizing in particular the finite-size corrections to the leading-order free energy and showing that they are indeed expressed as a sum on cycle contributions. Moreover, in the matching problem these topological contributions seem to be directly related to the finite-size corrections appearing in the fully connected case, a fact suggested in [17] that will be investigated in the present paper, where this sparse-dense correspondence will be numerically verified.

The paper is organized as follows. In section 2 we will introduce the MP in full generality, and we will recall some of the known results about its AOC and the corresponding finite-size corrections. We will describe the cavity approach to derive the asymptotic cost on a random regular graph, we will discuss how to compute, using the cavity method, the finite-size corrections, and we will compare our population dynamics results with the numerics. In section 3 we will introduce a useful auxiliary version of the problem, called the random fractional matching problem (FMP), and we will study it by means of the same techniques. In section 4 we will further relax some constraints adopted in our problem to study so-called 'loopy' fractional matching, giving the cavity equations for its solution both at the leading level and at the subleading order. In 
section 5 we will further comment on the $z \rightarrow N$ limit, where $z$ is the coordination of the Bethe lattice. Finally, in section 6 we will give our conclusions.

\section{The random-link matching problem}

The MP on a generic graph $G=(\mathcal{V} ; \mathcal{E})$ with vertex set $\mathcal{V}$ having cardinality $N=|\mathcal{V}|$, and edge set $\mathcal{E} \subseteq \mathcal{V} \times \mathcal{V}$ is defined as follows. For each edge $e \in \mathcal{E}$ of the graph we draw a random weight $w_{e} \in \mathbb{R}^{+}$distributed with a probability density function $\varrho(w)$, which we assume to have support over the positive real axis. We associate an occupancy variable $m_{e} \in\{0,1\}$ with each edge $e \in \mathcal{E}$, in such a way that $m_{e}=1$ if the edge is considered occupied, and $m_{e}=0$ otherwise. We search for the maximum cardinality matching that has minimum cost, i.e. for the set of values $M=\left\{m_{e}\right\}_{e}$ satisfying the constraints

$$
m_{e} \in\{0,1\}, \quad \sum_{e \rightarrow v} m_{e} \leqslant 1 \forall v \in \mathcal{V},
$$

that maximizes

$$
|M|:=\sum_{e} m_{e}
$$

and minimizes the cost:

$$
E_{G}[M]:=\frac{1}{|M|} \sum_{e} m_{e} w_{e}
$$

In equation (1) the sum runs over all edges incident to the vertex $v \in \mathcal{V}$. A matching is said to be perfect if $2|M|=N$; a perfect matching is not possible on a generic graph, unless the hypotheses of Tutte's theorem are fulfilled (see [1]). Note that assuming an even $N$ does not guarantee the existence of a perfect matching. For example, the following graph

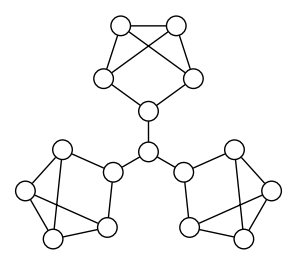

is the only connected regular graph with $N=16$ and coordination equal to 3 not allowing for a perfect matching. Given a graph $G$ and denoting by $\mathcal{M}(G)$ the set of all possible matching on $G$ of maximal cardinality, one might be interested in the AOC, i.e.

$$
E_{G}:=\mathbb{E}\left[\min _{M \in \mathcal{M}(G)} E_{G}[M]\right]
$$

where the notation $\mathbb{E}[\bullet]$ represents the average over the disorder. In 1985, Mézard and Parisi [13] considered the case $G=K_{N}$, a complete graph with $|\mathcal{V}|=N$ vertices. Using both the replica approach [13] and the cavity method [14], Mézard and Parisi were able to evaluate the AOC in this problem for $N \rightarrow+\infty$, finding that, if $\lim _{w \rightarrow 0} \varrho(w)=\frac{2}{N}$, 


$$
E_{\infty}:=\lim _{N \rightarrow+\infty} E_{K_{N}}=\frac{\zeta(2)}{2},
$$

where $\zeta(z)$ is the Riemann zeta function. The study of the finite-size corrections to this asymptotic value requires some effort and it was performed in [15-17]. In particular, adopting a weight distribution

$$
\varrho_{N}(w)=\frac{2}{N} \mathrm{e}^{-\frac{2 w}{N}} \theta(w)
$$

with a $\theta(\bullet)$ Heaviside function, we have

$$
E_{K_{N}}=\frac{\zeta(2)}{2}+\frac{\zeta(2)}{2 N}+\frac{2}{N} \sum_{\substack{p \geqslant 3 \\ \text { odd }}} \frac{I_{p}}{p}+o\left(\frac{1}{N}\right)=\frac{\zeta(2)}{2}-\frac{0.0674(1)}{N}+o\left(\frac{1}{N}\right),
$$

where, denoting $x_{p+1} \equiv x_{1}$,

$$
I_{p}:=\int_{1}^{\infty} \frac{\mathrm{d} y}{2 y} \prod_{i=1}^{p} \int_{0}^{1} \frac{\theta\left(1-x_{i} x_{i+1}\right) \mathrm{d} x_{i}}{x_{i}+y}=\frac{1}{p}+o\left(\frac{1}{p}\right) .
$$

In [29] it was shown that, using the weight distribution in equation (6), the subsubleading corrections scale in an anomalous way, i.e. as $N^{-\frac{3}{2}}$, and not as $N^{-2}$ as one might expect. Moreover, in [17] it was observed that, if the constraints are relaxed and any value $0 \leqslant m_{e} \leqslant 1$ is allowed, cycles appear in the optimal solution, and, at the same time, both the sum contribution in equation (7) and the anomalous correction disappear. If, finally, one allows a vertex to 'self-match', the $\frac{1}{N}$ corrections disappear altogether. These facts suggest that the $\frac{1}{N}$ corrections in equation (7) are due to cycles in the graph, and the sum in equation (7) runs over contributions corresponding to cycles of different lengths $p$. This hypothesis also justifies the presence of the $O\left(N^{-\frac{3}{2}}\right)$ correction that naturally appears imposing a cut-off to the sum in equation (7), taking into account the fact that the possible length of cycles is bounded for finite $N$ [29]. Surprisingly enough, if instead of $K_{N}$ a bipartite complete graph $K_{N, N}$ is considered, the form of the finite-size corrections strongly simplifies. In the bipartite complete graph, $2 N$ vertices are divided into two disjoint sets having the same cardinality $N$, and an edge is present between two vertices if and only if they belong to different sets. Assuming the same weight distribution given in equation (6), it is possible to prove that the AOC in this case is given by [30-32]

$$
E_{K_{N, N}}=\sum_{i=1}^{N} \frac{1}{i^{2}}=\zeta(2)-\frac{1}{N}+o\left(\frac{1}{N}\right) .
$$

Note that the expression holds for any value of $N$. The simplification, and the absence of anomalous correction terms, suggests once again that cycles are indeed relevant for finite-size correction (only even cycles appear in bipartite graphs).

The role of topological structures, e.g. paths, cycles and loops, in finite-size corrections clearly emerged in the study of disordered spin systems on random graphs [26, 33]. In these cases, the cavity approach made clear the correspondence inferred above, 
and the corrections were actually evaluated by studying finite-size fluctuations in the graph topology with respect to the asymptotic infinite tree structure. For the same reasons, in the present paper, we will consider the MP on a random regular graph ensemble $\mathbb{G}_{\mathrm{RRG}}(N, z)$, and we will study both the AOC in the thermodynamic limit and its finite-size corrections. We consider a uniform measure ${ }^{3}$ over all graphs with $N$ vertices whose coordination is exactly $z$. For fixed $z \geqslant 3$, an element of this ensemble $\mathbb{G}_{\mathrm{RRG}}(N, z)$ admits almost always a perfect matching if $z N$ is even [36]. Random regular graphs have cycles of typical length $\frac{\ln N}{\ln z}$ for $N \gg 1$ and in the $N \rightarrow+\infty$ limit at fixed $z$, the Cayley tree of coordination $z$ is recovered. On the other hand, for $z=N-1$ a complete graph $K_{N}$ is obtained. Random regular graphs are therefore excellent candidates for the study of both corrections due to cycles and finite-coordination effects. On top of the random topology, we generate a random weight for each edge of the graph with distribution $\varrho(w)$, and we search for the optimal matching cost on the obtained weighted graph. The AOC is obtained by averaging over both the weights and the graph topology realizations. In our problem, therefore, two sources of disorder appear, namely the topological disorder, due to the fact that the graph is randomly extracted from the $\mathbb{G}_{\mathrm{RRG}}(N, z)$ ensemble, and the link-weight disorder, due to the fact that the weights are i.i.d. random variables distributed with probability density function $\varrho(w)$. The AOC density is evaluated by averaging over both these sources of disorder:

$$
E_{z}(N):=\mathbb{E}\left[\min _{M \in \mathcal{M}(G)} E_{G}[M]\right]=E_{z}+\frac{E_{z}^{(1)}}{N}+o\left(\frac{1}{N}\right),
$$

where $\lim _{N \rightarrow+\infty} E_{z}(N)=E_{z}$. In the following, we will assume an exponential probability density:

$$
\varrho_{z}(w):=\frac{2}{z} \mathrm{e}^{-\frac{2 w}{z}} \theta(w)
$$

\subsection{The asymptotic cost}

The cavity method is a natural candidate for the study of random optimization on sparse graphs [11, 37]. In the particular case of the MP, we start writing down a partition function associated with our problem on a graph $G=(\mathcal{V} ; \mathcal{E})$ extracted from $\mathbb{G}_{\mathrm{RRG}}(N, z)$, as

$$
Z(\beta):=\sum_{\left\{m_{e}=0,1\right\}} \prod_{v \in \mathcal{V}}\left[\mathbb{I}\left(\sum_{e \rightarrow v} m_{e} \leqslant 1\right) \mathrm{e}^{-\gamma\left(1-\sum_{e \rightarrow v} m_{e}\right)}\right] \mathrm{e}^{-\frac{\beta N}{2} E_{G}[M]} .
$$

The indicator function $\mathbb{I}(s)$ of a statement $s$ is such that $\mathbb{I}(s)=1$ if $s$ is true, and zero otherwise. Observe that we have introduced the Lagrange multiplier $\gamma$ to impose the fact that the optimal matching is perfect. The AOC can be therefore obtained by

\footnotetext{
${ }^{3}$ The measure is uniform over all labeled graphs [34]. Another possibility is to consider a uniform measure over all non-isomorphic regular graphs with $N$ vertices and coordination $z$. However it can be proved that the two definitions are equivalent in the thermodynamic limit [35].
} 


$$
E_{z}=-\lim _{N \rightarrow+\infty} \lim _{\beta \rightarrow+\infty} \lim _{\gamma \rightarrow+\infty} \frac{2 \mathbb{E}[\ln Z(\beta)]}{\beta N} .
$$

The variables of our problem are the quantities $m_{e} \in\{0,1\}$ defined on the edges of our weighted graph $G$. They have to satisfy the constraints in equation (1); moreover, a weight $\exp \left[-\gamma\left(1-\sum_{e \rightarrow v} m_{e}\right)\right]$ appears in the partition function in equation (11) for each vertex of the graph $G$. It is well known that we can associate with the partition function a bipartite graph, called a factor graph, involving two types of nodes, called variable nodes and functional nodes. Variable nodes correspond to the edges of $G$, and they are linked to functional nodes representing the constraint in equation (1) and the reweighting that asymptotically imposes the perfect matching:

$$
\bigcap_{0}-\equiv \mathbb{I}\left[\sum_{e \rightarrow v} m_{e} \leq 1\right] \mathrm{e}^{-\gamma\left(1-\sum_{e \rightarrow v} m_{e}\right)}
$$

Each functional node of this kind is linked to the variable nodes involved in the constraint, and uniquely corresponds to a vertex in the graph $G$. Moreover, each variable node is linked to an additional functional node corresponding to the $\mathrm{e}^{-\beta m_{e} w_{e}}$ reweighting ${ }^{4}$ :

$$
\underset{e}{C_{e}} \equiv \mathrm{e}^{-\beta m_{e} w_{e}} .
$$

We can finally construct our factor graph following the rules above starting from $G$. Pictorially,

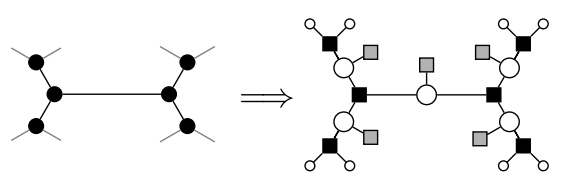

For each edge $e=(i, j)$ in the graph $G$ we can define an 'incoming' message $\mu^{i \rightarrow e}$ from the functional node $i$ to the variable node $e$ as the marginal probability distribution of the variable $m_{e}$ obtained by removing any other edge with endpoint $e$ except $(i, e)$ in the factor graph. Pictorially,

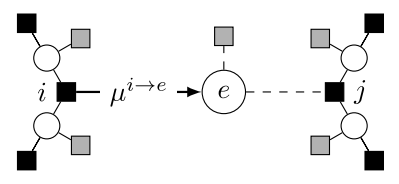

For $N \gg 1$, the tree-like structure of $G$, inherited by the factor graph, allows us to write a recurrence equation for $\mu^{i \rightarrow e}$, namely

$\mu^{i \rightarrow e}\left(m_{e}\right) \propto \sum_{\substack{\left\{m_{\hat{e}}\right\} \\ \hat{e} \neq e}} \mathbb{I}\left(\sum_{\hat{e} \rightarrow i} m_{\hat{e}} \leqslant 1\right) \exp \left[-\gamma\left(1-\sum_{\hat{e} \rightarrow i} m_{\hat{e}}\right)\right] \prod_{\substack{\hat{e} \rightarrow i \\ \hat{e}=(k, i) \neq e}} \mathrm{e}^{-\beta m_{\hat{e}} w_{\hat{e}}} \mu^{k \rightarrow \hat{e}}\left(m_{\hat{e}}\right)$.

\footnotetext{
${ }^{4}$ Here we assume that $\eta:=2 M N^{-1}$ is exactly equal to 1 . As mentioned before, almost surely $\eta=1$ for $N \rightarrow+\infty$ [38]. The possible presence of $O\left(\frac{1}{N}\right)$ corrections in $\eta$ to this asymptotic limit might give additional $O\left(\frac{1}{N}\right)$ finite-size corrections to the asymptotic cost. However, with the graph sizes considered in this paper, we never find connected regular graphs without perfect matching in around $10^{7}$ Monte Carlo steps, so we assume finite- $N$ corrections to $\eta=1$ to be of higher order.
} 
In the expression above, we make the assumption that the marginal distribution corresponding to edges $i \rightarrow \hat{e}$ such that $\hat{e} \neq e$ factorizes: this assumption is exact on trees [11]. We can introduce a cavity field $h^{i \rightarrow e}$ that parametrizes the message $\mu^{i \rightarrow e}\left(m_{e}\right)$ as

$$
h^{i \rightarrow e}:=-\frac{1}{\beta} \ln \frac{\mu^{i \rightarrow e}(0)}{\mu^{i \rightarrow e}(1)}=-\frac{1}{\beta} \ln \left[\mathrm{e}^{-\gamma}+\sum_{\substack{\hat{\hat{e} \rightarrow i} \\ \hat{e}=(k, i) \neq e}} \mathrm{e}^{-\beta\left(w_{\hat{e}}-h^{k \rightarrow \hat{e}}\right)}\right] .
$$

The marginal distribution of the variable $m_{e}$ is therefore parametrized as $\mu\left(m_{e}\right) \propto \exp \left[-\beta\left(w_{e}-h^{i \rightarrow e}-h^{j \rightarrow e}\right) m_{e}\right]$. Equation (14) implies, for $\beta \rightarrow+\infty$ and $\gamma \rightarrow+\infty$

$$
h^{i \rightarrow e}=\min _{\substack{\hat{e} \rightarrow i \\ \hat{e}=(k, i) \neq e}}\left(w_{e}-h^{k \rightarrow \hat{e}}\right)
$$

where the minimum runs over $z-1$ edges incident to $j$. Node $i$ is then matched using edge $e^{*}$ such that

$$
e^{*}=\arg \min _{\substack{\hat{e} \rightarrow i \\ \hat{e}=(k, i) \neq e}}\left(w_{\hat{e}}-h^{k \rightarrow \hat{e}}\right)
$$

or, equivalently, edge $e=(i, j)$ is occupied if $w_{e} \leqslant h^{i \rightarrow e}+h^{j \rightarrow e}$. The equation above can be used to solve a specific instance of the problem by means of a message-passing algorithm [6, 11, 23]. Being interested in the AOC, however, we will infer from equation (15) the distributional equation

$$
h \stackrel{d}{=} \min _{1 \leqslant e \leqslant z-1}\left(w_{e}-h_{e}\right)
$$

where the equality holds in the distributional sense, the $z-1$ quantities $\left\{w_{e}\right\}_{e}$ follow the distribution $\varrho_{z}(w)$ of the edge weights and the $z-1$ cavity fields $\left\{h_{e}\right\}_{e}$ have the same distribution $p_{z}(h)$ of $h$. By means of a population dynamics algorithm, and using equation (17), the distribution of the cavity fields $p_{z}(h)$ can be numerically evaluated. The AOC for $N \rightarrow+\infty$ is then

$$
E_{z}=z \int_{0}^{+\infty} \mathrm{d} w w \varrho_{z}(w) \iint \mathrm{d} h_{1} \mathrm{~d} h_{2} \theta\left(h_{1}+h_{2}-w\right) p_{z}\left(h_{1}\right) p_{z}\left(h_{2}\right) .
$$

Denoting

$$
\Phi_{z}(x):=\int_{x}^{\infty} p_{z}(h) \mathrm{d} h,
$$

from equation (17) we can write

$$
\Phi_{z}(x)=\left(1-\mathbb{E}\left[\Phi_{z}(w-x)\right]\right)^{z-1},
$$

where the average is taken over the variable $w$. In the appendix we solve this equation up to $o\left(\frac{1}{z}\right)$ corrections to estimate the finite- $z$ corrections to the asymptotic AOC, finding that, in the large- $z$ expansion of $E_{z}$, the $\frac{1}{z}$ term is identically zero,

$$
E_{z}=\frac{\zeta(2)}{2}+O\left(\frac{1}{z^{2}}\right)
$$


implying that

$$
\lim _{z \rightarrow+\infty} \lim _{N \rightarrow+\infty}\left(E_{K_{N}}-E_{z}(N)\right)=0 .
$$

This nontrivial sparse-dense correspondence is of the type discussed in [39]. The obtained AOC corresponds to the cost on the infinite tree, and finite-size corrections to $E_{z}$ are out of the reach of the approach above. The formula above holds for both the $\mathrm{MP}$ and the assignment problem (AP) that we will discuss below, due to the fact that the two problems are actually the same on trees, and they only differ at the level of finite-size corrections.

\subsection{Finite-size corrections}

As anticipated, finite-size corrections are not directly accessible through the straightforward calculation given in the previous section; however, following [26, 33], we expect that they can be expressed in terms of contributions due to the topological structures that appear in the graph with $O\left(\frac{1}{N}\right)$ density. In particular, in the case of random regular graphs we expect cycles to give a fundamental contribution ${ }^{5}$. Indeed, the number of cycles of length $\ell$ in an infinite random regular graph of coordination $z$ is a Poisson variable [41] with mean

$$
\bar{n}_{\ell}(z)=\frac{(z-1)^{\ell}}{2 \ell} \text {. }
$$

The fact that the density of cycles is infinitesimal for $N \rightarrow+\infty$ suggests that the contribution $\phi_{\ell}(z)$ of each cycle of length $\ell$ can be considered as independent from the others, due to the increasingly large distance of cycles for $N \gg 1$, and therefore we can evaluate it as the contribution of a single cycle embedded in an infinite tree. On the basis of these observations, we expect $E_{z}^{(1)}$ to have the form

$$
E_{z}^{(1)}=v_{z}+\sum_{\ell=3}^{\infty} \bar{n}_{\ell}(z) \phi_{\ell}(z),
$$

where $v_{z}$ is cycle-independent ${ }^{6}$. The cycle contribution $\phi_{\ell}(z)$ can be therefore evaluated using the cavity approach as

$$
\phi_{\ell}(z)=\lim _{\beta \rightarrow+\infty} \mathbb{E}\left[-\frac{1}{\beta} \ln \frac{Z_{\ell}(\beta)}{Z_{\mathrm{T}}(\beta)}\right]
$$

where $Z_{\mathrm{T}}(\beta)$ is the partition function of the infinite tree with coordination $z$ and $Z_{\ell}(\beta)$ is the partition function for a $z$-regular graph that differs from the infinite tree because of the presence of a single cycle of length $\ell$. The average is taken on the weights appearing on the edges. It is important to note that both graphs can be obtained from the same object, i.e. a 'cavity' tree with $2 \ell+2$ 'cavity variable nodes',

\footnotetext{
${ }^{5}$ As noted in [26], an additional contribution, due to chains in the graph, has to be considered in general, e.g. in Erdős-Rényi random graphs. In the random regular case, this contribution is absent due to the fact that there are no fluctuations in vertex connectivity [33].

${ }^{6}$ The presence of an additional constant $v_{z}$ might depend on the choice of weight distribution function $\varrho_{z}(w)$ and/ or possible symmetries of the problem $[16,17]$.
} 


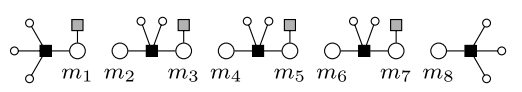

Because of the tree-like structure, the partition function of such a cavity factor graph conditioned to a given configuration $\left(m_{1}, \ldots, m_{2 \ell+2}\right)$ of the cavity variable nodes factorizes in $\ell+2$ contributions. We can also write the messages corresponding to each contribution. The marginal probability of these cavity variables can be written as

$$
\begin{aligned}
& \underset{\delta_{i}}{\stackrel{\mathcal{L}_{e}}{\circ}}=\frac{Z_{1}\left(m_{e}, \beta\right)}{Z_{1}(\beta)}=\mu^{i \rightarrow e}\left(m_{e}\right) \text {, } \\
& \underset{\delta_{i}}{\oint_{e}^{0}}=\frac{Z_{1}^{\prime}\left(m_{e}, \beta\right)}{Z_{1}^{\prime}(\beta)} \propto \mathrm{e}^{-\beta m_{e} w_{e}} \mu^{i \rightarrow e}\left(m_{e}\right), \\
& \overbrace{e} \underbrace{\mathcal{O}}_{i} \underbrace{0}_{\tilde{e}}=\frac{Z_{2}\left(m_{e}, m_{\tilde{e}}, \beta\right)}{Z_{2}(\beta)} \equiv \hat{\mu}^{i \rightarrow e, \tilde{e}}\left(m_{e}, m_{\tilde{e}}\right) .
\end{aligned}
$$

Here $Z_{2}(\beta)$ is the partition function of a graph containing two cavity variables, whereas $Z_{2}\left(m_{1}, m_{2}, \beta\right)$ is the partition function of the same graph obtained by constraining the cavity variables to have values $m_{1}$ and $m_{2}$. Similar notation holds for $Z_{1}$ and $Z_{1}^{\prime}$. The marginals defined in equations (26a) and (26b) are exactly the same messages in equation (13). The marginal in equation (26c) satisfies instead the equation

$\hat{\mu}^{i \rightarrow e, \tilde{e}}\left(m_{e}, m_{\tilde{e}}\right) \propto \mathrm{e}^{-\beta m_{\tilde{e}} w_{\tilde{e}}} \sum_{\left\{m_{\hat{e}}\right\} \hat{e} \neq e, \tilde{e}} \mathbb{I}\left(\sum_{\hat{e} \rightarrow i} m_{\hat{e}}=1\right) \prod_{\substack{\hat{e} \rightarrow i \\ \hat{e}=(k, i) \neq e, \tilde{e}}} \mathrm{e}^{-\beta m_{\hat{e}} w_{\hat{e}}} \mu^{k \rightarrow \hat{e}}\left(m_{\hat{e}}\right)$.

This marginal is such that $\hat{\mu}^{i \rightarrow e, \tilde{e}}(1,1)=0$. The normalization constraint leaves us two parameters to parametrize the distribution; moreover

$$
-\frac{1}{\beta} \ln \frac{\hat{\mu}^{i \rightarrow e, \tilde{e}}(0,1)}{\hat{\mu}^{i \rightarrow e, \tilde{e}}(1,0)}=w_{\tilde{e}} .
$$

We can therefore parametrize the distribution with one parameter only, writing

$$
\hat{\mu}^{i \rightarrow e, \tilde{e}}\left(m_{e}, m_{\tilde{e}}\right) \propto \mathrm{e}^{-\beta m_{\tilde{e}} w_{\tilde{e}}+\beta \hat{h}^{i \rightarrow e, \tilde{e}}\left(1-m_{e} m_{\tilde{e}}\right)} \mathbb{I}\left(m_{e} m_{\tilde{e}} \neq 1\right) .
$$

The partition function of the entire object is therefore $Z_{\text {cav }}(\beta)=Z_{1}(\beta) Z_{1}^{\prime}(\beta) Z_{2}^{\ell}(\beta)$. On the other hand, from the cavity graph we can reconstruct an original tree identifying $m_{2 i-1}$ and $m_{2 i}$ for $i=1, \ldots, \ell+1$, obtaining the ratio between the partition function of the tree $Z_{\mathrm{T}}(\beta)$ and $Z_{\text {cav }}(\beta)$,

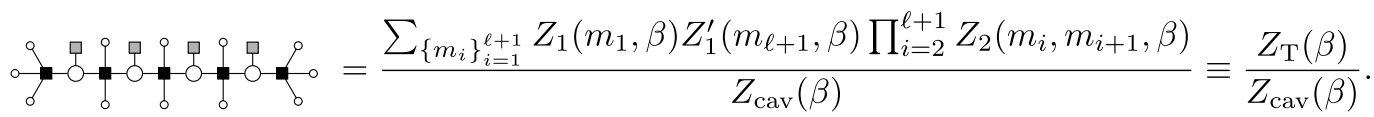

Similarly, in order to get $Z_{\ell}(\beta) Z_{\text {cav }}^{-1}(\beta)$, we can construct from the cavity graph a topology containing a loop

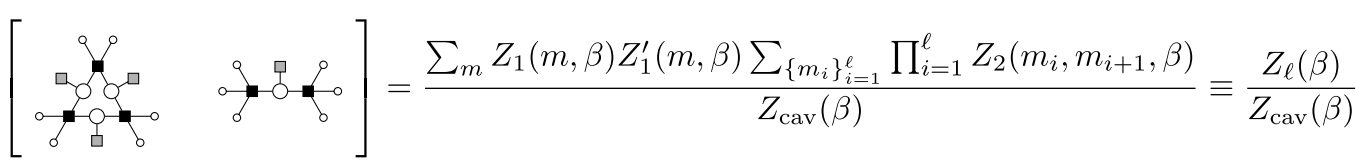


(where in the product $m_{\ell+1} \equiv m_{1}$ ). Using equations (26), it can be easily shown that the specific contribution of the cycle can be expressed as a trace of products of random matrices as follows:

$$
Z_{\ell}^{\mathrm{c}}(\beta):=\sum_{\left\{m_{i}\right\}_{i=1}^{\ell}} \prod_{i=1}^{\ell} \frac{Z_{2}\left(m_{i}, m_{i+1}, \beta\right)}{Z_{2}(\beta)}=\operatorname{tr} \prod_{i=1}^{\ell} \mathbf{T}_{i}, \quad \mathbf{T}_{i}:=\left(\begin{array}{cc}
\mathrm{e}^{\beta \hat{h}_{i}} & 1 \\
\mathrm{e}^{-\beta w_{i}} & 0
\end{array}\right),
$$

where $\hat{h}_{i}$ denotes the cavity fields entering the cycle. Note that this structure is reminiscent of equation $(7 b)$. The study of the spectral properties of the transfer matrix $\mathbf{T}$, performed using the replica trick, can provide an estimate of the large $\ell$ behavior $\phi_{\ell}$ $[26,33]$. Due to the fact that in our problem the variables live on the edges, and not on the vertices of the graph, from the factor graph point of view, the cycle is connected to the rest of the graph through its functional nodes (and not through its variable nodes). More importantly, the messages entering the cycle are determined by the infinite treelike structure. Similarly, a chain contribution can be written as

$$
Z_{\ell}^{\mathrm{a}}(\beta):=\sum_{\left\{m_{i}\right\}_{i=1}^{\ell}} \frac{Z_{1}\left(m_{1}, \beta\right)}{Z_{1}(\beta)} \frac{Z_{1}^{\prime}\left(m_{\ell}, \beta\right)}{Z_{1}^{\prime}(\beta)} \prod_{i=2}^{\ell-1} \frac{Z_{2}\left(m_{i}, m_{i+1}, \beta\right)}{Z_{2}(\beta)}=\mathbf{u}_{1} \cdot\left(\prod_{i=2}^{\ell-1} \mathbf{T}_{i}\right) \cdot \mathbf{u}_{\ell}
$$

where $\mathbf{u}_{i}=\left(\mathrm{e}^{\beta h_{i}}, \mathrm{e}^{-\beta w_{i}}\right)$.

In the $\beta \rightarrow+\infty$ limit, the equations strongly simplify. First observe that, from equations (27) and (29) we obtain

$$
\hat{h}^{i \rightarrow e, \tilde{e}}=\min _{\substack{\hat{e} \rightarrow i \\ \hat{e} \neq e, \tilde{e}}}\left(w_{\hat{e}}-h^{k \rightarrow i}\right) .
$$

The field $\hat{h}$ corresponds to the outgoing cavity field from a functional node having $z-2$ neighbors. In the $\beta \rightarrow \infty$ limit, the fields $\hat{h}$ satisfy the distributional equation

$$
\hat{h} \stackrel{d}{=} \min _{1 \leqslant e \leqslant z-2}\left(w_{e}-h_{e}\right) \text {. }
$$

We can define the chain cost $\phi_{\ell}^{\mathrm{a}}$ and the cycle $\operatorname{cost} \phi_{\ell}^{\mathrm{c}}$ as

$$
\phi_{\ell}^{\mathrm{a}}(z):=-\lim _{\beta \rightarrow+\infty} \frac{\mathbb{E}\left[\ln Z_{\ell}^{\mathrm{a}}(\beta)\right]}{\beta}, \quad \phi_{\ell}^{\mathrm{c}}(z):=-\lim _{\beta \rightarrow+\infty} \frac{\mathbb{E}\left[\ln Z_{\ell}^{\mathrm{c}}(\beta)\right]}{\beta},
$$

and write an equation that is similar to the one appearing in the cycle expansion of finite-size corrections of spin-glass systems on random graphs [26, 33]

$$
\phi_{\ell}(z)=\phi_{\ell}^{\mathrm{c}}(z)-\left(\phi_{\ell+2}^{\mathrm{a}}(z)-\phi_{2}^{\mathrm{a}}(z)\right) .
$$

The $\beta \rightarrow+\infty$ limit can be numerically evaluated by considering, for each cycle or chain of length $\ell$, all possible ways of occupying it, and averaging over the minimum-cost configurations: each internal edge contributes with its weight, and each external edge entering the matching gives (minus) the corresponding cavity field. For example, for $\ell=3$, 


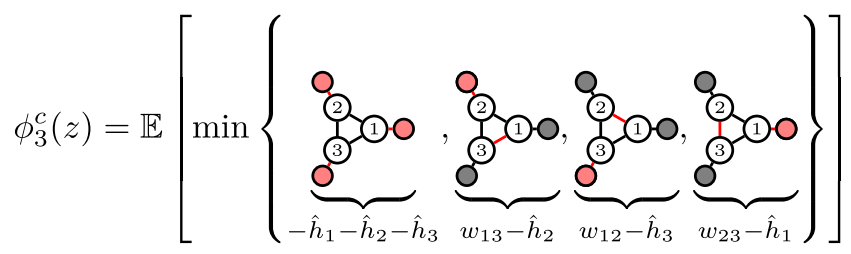

For each instance, we have three incoming cavity fields and three weights associated with the cycle edges. In this case there are four possible configurations: one corresponding to the occupation of external lines only, involving three incoming cavity fields $\hat{h}$ (larger blobs), and three different configurations in which an edge belonging to the loop is occupied, plus an external edge contributing with its cavity field. The cost is obtained by averaging over the minimum-cost configurations.

\subsection{Numerical results}

Let us start discussing the numerical results about the AOC of the MP and the general properties of its scaling in the MP on random regular graphs. The results of the cavity computation for different values of $z$ are given in figure 1(a) and in table 1. As expected, with the adopted convention $\lim _{z \rightarrow+\infty} E_{z}=E_{\infty}$. The cavity estimation has been compared with the AOC obtained by solving the problem on random regular graphs with $3 \leqslant z \leqslant 15$ extracted from $\mathbb{G}_{\mathrm{RRG}}(N, z)$ and extrapolating for $N \rightarrow+\infty$. For all the investigated values of $z$, the scaling of the AOC is in agreement with the ansatz

$$
E_{z}(N)=E_{z}+\frac{E_{z}^{(1)}}{N}+\frac{E_{z}^{\left(\frac{3}{2}\right)}}{N^{\frac{3}{2}}}+\frac{E_{z}^{(2)}}{N^{2}}+o\left(\frac{1}{N^{2}}\right),
$$

showing the same anomalous correction appearing in the MP on complete graphs [17] (see figure 1(b)). The numerical estimations obtained by extrapolating the asymptotic AOC from the finite- $N$ results are in perfect agreement with the cavity prediction for $E_{z}$. Moreover, we observe that for large values of $z$, the coefficient of the $\frac{1}{z}$ term in the large $z$ expansion of $E_{z}$ is compatible with zero, as expected from the analytical calculation. Assuming a quadratic dependence on $\frac{1}{z}$, we find

$$
E_{z}=\frac{\zeta(2)}{2}+\frac{0.057(1)}{z^{2}}+o\left(\frac{1}{z^{2}}\right)
$$

To analyze now the finite-size correction $E_{z}^{(1)}$, let us start focusing on the singlecycle contributions. The quantity $\phi_{\ell}(z)$ has been first evaluated by means of a population dynamics algorithm for different values of $\ell$ and $z$, using equation (25), and then compared with the results obtained numerically by solving the MP on random regular graphs. In particular, the contribution of a cycle of length $\ell$ to the optimal cost can be evaluated numerically using a Markov chain of length $T$ of regular graphs of size $N$, $\left\{G_{t}\right\}_{t=1}^{T}$, such that $G_{t+1}$ is obtained from $G_{t}$ by means of a single-edge swapping. Let $E_{t}$ be the optimal cost corresponding to element $G_{t}$ of the chain, and let $n_{\ell}^{t}$ be the number of cycles of length $\ell$ in graph $G_{t}$. The average cost shift due to the appearance of a new cycle of length $\ell$ in a graph having $n_{\ell}$ cycles of length $\ell$ is 
Random-link matching problems on random regular graphs

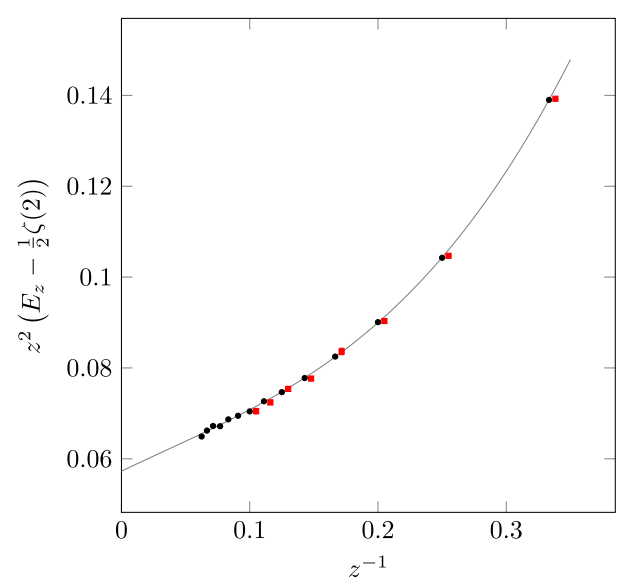

(a)

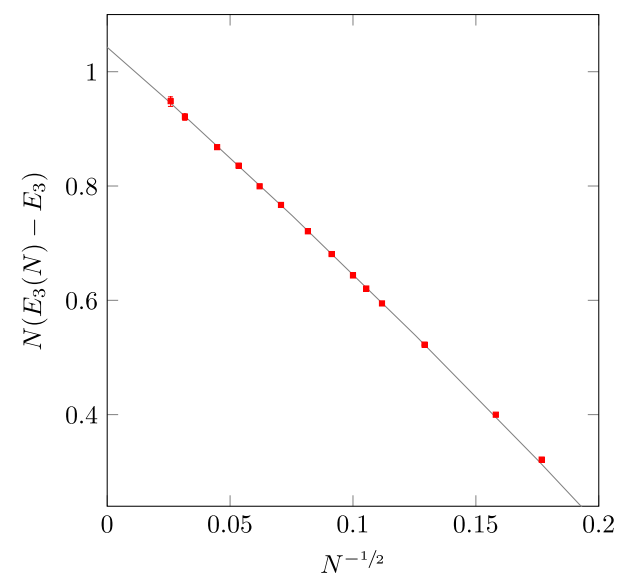

(b)

Figure 1. Numerical and cavity results for the AOC in the MP on random regular graphs. The cavity results have been obtained with a population of $10^{7}$ fields. For the numerical evaluation of the AOC of the random-link problems, we make use of the Lemon Graph Library [40]. We mention here that, to improve the estimate of the AOC, we adopt a simple trick. Let us suppose that we want to estimate the average $\langle A\rangle$ of some random scalar quantity $A$, and that we exactly know the average $\langle B\rangle$ of a second scalar quantity $B$ that is positively correlated with $A$. Then, $\operatorname{Var}(A-B)=\operatorname{Var}(A)+\operatorname{Var}(B)-2\langle A B\rangle+2\langle A\rangle\langle B\rangle$. It is possible therefore, depending on the choice of $B$, that $\operatorname{Var}(A)>\operatorname{Var}(A-B)$. In this case, evaluating $\langle A\rangle$ as $\langle A-B\rangle+\langle B\rangle$ can be less noisy. We follow this approach for the AOC, choosing for $B$ the heuristic cost given by selecting for each node the lowest-weight incident edge. (a) AOC of the MP on random regular graphs with $z \geqslant 3$ with the exponential weight distribution given in equation (10). The cavity results (black circles) are compared with the AOC (red squares, shifted by $10^{-3}$ in the $x$ direction for the sake of clarity) obtained by solving the problem on random regular graphs of size $N=10^{5}$. The continuous line is obtained from a cubic fit. (b) Scaling of the finite-size correction for $z=3$ for the MP. See also table 1 . The continuous line is a quadratic fit in $\frac{1}{\sqrt{N}}$.

$$
\phi_{\ell}(z ; N, T, n):=\mathbb{E}\left[\frac{\sum_{t=1}^{T}\left(E_{t+1}-E_{t}\right) \mathbb{I}\left(n_{\ell}^{t+1}=n_{\ell}^{t}+1\right) \mathbb{I}\left(n_{\ell}^{t}=n\right)}{\sum_{t=1}^{T} \mathbb{I}\left(n_{\ell}^{t+1}=n_{\ell}^{t}+1\right) \mathbb{I}\left(n_{\ell}^{t}=n\right)}\right] .
$$

The contribution $\phi_{\ell}(z)$ is obtained numerically by extrapolating the previous quantity in the limit of large-size and long Markov chains:

$$
\phi_{\ell}(z)=\lim _{\substack{T \rightarrow+\infty \\ N \rightarrow+\infty}} \phi_{\ell}(z ; N, T, n) .
$$

Note that the right-hand side of the previous equation does not depend on $n$, since the density of cycles of a given length vanishes in the large-size limit.

By both cavity and direct estimations, we obtain the fact that $\left|\phi_{\ell}(z)\right|$ decreases both in $z$ and in $\ell$. The numerical estimations obtained for $z=3$ and $z=4$ and $\ell \leqslant 5$ are found to be in agreement with the cavity predictions for small values of $\ell$ (see figure 2(b) and table 2). For larger values of $\ell, \ell^{-1} \phi_{\ell}$ becomes too small and we are not able to evaluate it numerically by solving the MP with statistical significance. On the 
Table 1. Summary table of the numerical estimates for the AOC and its finite-size corrections for the MP and its variants discussed in the paper for $z=3$ and $z=4$. For the details about the numerical simulations and the population dynamics algorithm, see the label of figure 1.

\begin{tabular}{llll}
\hline & & $z=3$ & $z=4$ \\
\hline Cavity & $E_{z}=E_{z}^{\mathrm{A}}=E_{z}^{\mathrm{F}}$ & $0.8379047(6)$ & $0.828967(2)$ \\
& $E_{z}^{\mathrm{L}}$ & $0.6096816(3)$ & $0.65342(1)$ \\
\hline $\mathrm{MP}$ & $E_{z}$ & $0.837903(3)$ & $0.82896(1)$ \\
& $E_{z}^{(1)}$ & $1.045(3)$ & $0.681(3)$ \\
& $E_{z}^{\left(\frac{3}{2}\right)}$ & $-3.88(7)$ & $-2.72(4)$ \\
\hline $\mathrm{AP}$ & $E_{z}^{\mathrm{A}}$ & $0.83786(7)$ & $0.828962(7)$ \\
& $E_{z}^{\mathrm{A},(1)}$ & $-1.5771(3)$ & $-1.313(2)$ \\
& $E_{z}^{\mathrm{A},(2)}$ & $7.12(6)$ & $4.2(1)$ \\
\hline $\mathrm{FMP}$ & $E_{z}^{\mathrm{F}}$ & $0.837903(5)$ & $0.828970(1)$ \\
& $E_{z}^{\mathrm{F},(1)}$ & $-0.03169(6)$ & $-0.0144(3)$ \\
& $E_{z}^{\mathrm{F},(2)}$ & $-5.60(1)$ & $-3.54(2)$ \\
\hline $\mathrm{LMP}$ & $E_{z}^{\mathrm{L}}$ & $0.609681(6)$ & $0.653423(1)$ \\
& $E_{z}^{\mathrm{L},(1)}$ & $0.0154(2)$ & $0.0160(1)$ \\
\hline
\end{tabular}

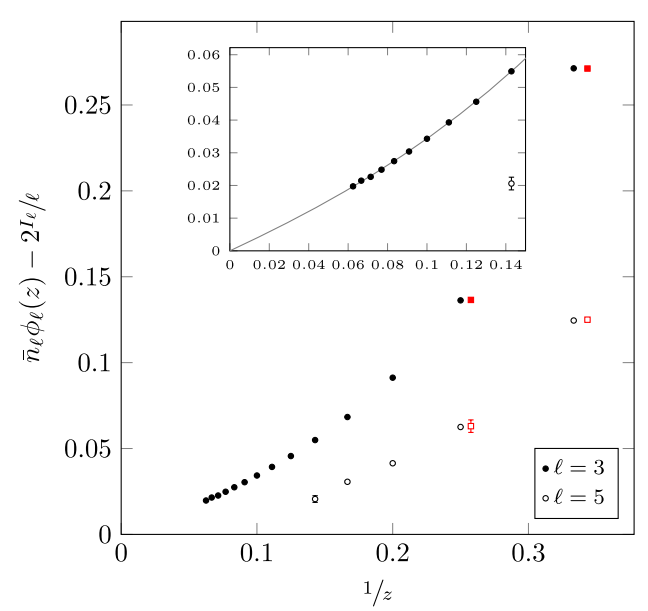

(a)

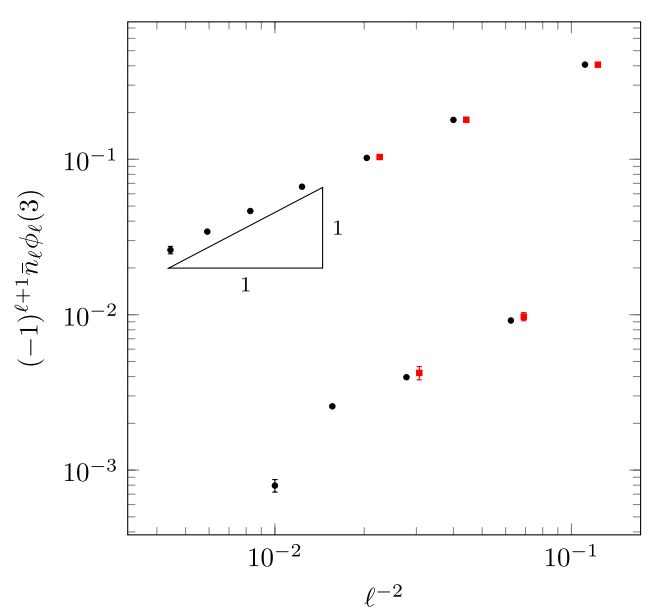

(b)

Figure 2. Cavity results for the cycle contributions in the MP on random regular graphs. Numerical results obtained by solving actual instances of the MP on random regular graphs have been plotted when available. The cavity results have been obtained using a population of $10^{8}$ fields. Cycle contributions for $\ell>3$ and $z \geqslant 4$ are very noisy due to the fact that we expect $\phi_{\ell}(z)$ to scale at least as $(z-1)^{-\ell}$ as $z \rightarrow+\infty$ and $\ell \rightarrow \infty$. (a) Average value of $\bar{n}_{\ell}(z) \phi_{\ell}(z)$ for $\ell=3,5$ and different values of $z$ (black circles), compared with the numerical estimations (red squares, slightly shifted in the $x$ direction for the sake of clarity). In the inset, zoomed-in view of the neighborhood of the origin. (b) Value of $\bar{n}_{\ell}(3) \phi_{\ell}(3)$ obtained by using the cavity method (black circles) and numerically solving the problem on the graph (red squares, slightly shifted in the $x$ direction for the sake of clarity). See also figure 3(b). 
Table 2. Summary table of the cavity estimates for $\bar{n}_{\ell}(z) \phi_{\ell}(z)$ for $z=3$ and $z=4$. The results have been obtained using a population of $10^{8}$ fields.

\begin{tabular}{|c|c|c|c|c|}
\hline \multirow[b]{2}{*}{$\bar{n}_{\ell} \phi_{\ell}(z)$} & \multicolumn{2}{|l|}{ MP } & \multirow{2}{*}{$\begin{array}{l}\text { FMP } \\
z=3\end{array}$} & \multirow{2}{*}{$\begin{array}{l}\text { LMP } \\
z=3\end{array}$} \\
\hline & $z=3$ & $z=4$ & & \\
\hline$\ell=3$ & $0.4067(1)$ & $0.2712(1)$ & $-0.005992(4)$ & $0.029108(4)$ \\
\hline$\ell=4$ & $-0.00918(2)$ & $-0.00360(4)$ & $-0.00918(2)$ & $-0.021584(8)$ \\
\hline$\ell=5$ & $0.1794(3)$ & $0.1174(1)$ & $-0.00354(1)$ & $0.01384(1)$ \\
\hline$\ell=6$ & $-0.003962(3)$ & $-0.0009(3)$ & $-0.003962(3)$ & $-0.00970(2)$ \\
\hline$\ell=7$ & $0.10214(5)$ & $0.065(9)$ & $-0.00192(6)$ & $0.00646(3)$ \\
\hline$\ell=8$ & $-0.00257(4)$ & $-0.03(1)$ & $-0.00257(4)$ & $-0.00449(5)$ \\
\hline$\ell=9$ & $0.06664(5)$ & $0.04(5)$ & $-0.0013(3)$ & $0.00297(5)$ \\
\hline$\ell=10$ & $-0.00082(5)$ & $0.1(1)^{\prime}$ & $-0.0008(6)$ & - \\
\hline$\ell=11$ & $0.0465(2)$ & - & - & - \\
\hline$\ell=13$ & $0.0344(4)$ & - & - & - \\
\hline$\ell=15$ & $0.026(1)$ & - & - & - \\
\hline
\end{tabular}

other hand, we can carry on our cavity estimation to larger cycle lengths. Remarkably, odd cycles and even cycles behave very differently, and the corresponding contributions lie on different curves (see figure 2(b)).

2.3.1. Odd-cycle contribution. If $\ell$ is odd, $\phi_{\ell}(z)$ is positive and, within the precision of our numerical results, we find that $\bar{n}_{\ell}(z) \phi_{\ell}(z) \rightarrow 2 \ell^{-1} I_{\ell}$ as $z \rightarrow+\infty$ for $\ell=3,5$ (see figure 2(a)). Here $I_{\ell}$ is the quantity appearing in equation (7): this result supports the hypothesis that the cycles' contributions in the MP on random regular graphs give indeed the sum in the finite-size corrections for the MP on the complete graph given in equation (7) for large $z$ [16], a fact a priori not obvious at all due to the fully connected nature of the latter problem. Moreover, at fixed $z$ and odd $\ell$, we find that $\bar{n}_{\ell}(z) \phi_{\ell}(z)$ scales as $\ell^{-2}$ for large values of $\ell$. This scaling justifies the presence of the anomalous $N^{-\frac{3}{2}}$ correction [29]. Indeed, let us assume that we have a path on the graph of length $k$ arriving at a certain site: the probability that $r$ of the $z-1$ outgoing link goes to one of the nodes already occupied by the path is $\left(\begin{array}{c}z-1 \\ r\end{array}\right)\left(\frac{k}{N}\right)^{r}\left(1-\frac{k}{N}\right)^{z-1-r}$. The probability of choosing one of these neighbors is $\frac{r}{z-1}$ : from this, it can be seen that the total probability of self-intersecting at step $k$ is equal to $\frac{k}{N}$. For an object of final length $\ell$ therefore we have a total probability of self-intersection that scales as $\frac{\ell^{2}}{N}$, which is of order one for $\ell \sim \sqrt{N}$. This suggests that we should impose a cut-off to the sum in equation (24) of order $\sqrt{N}$. But, if the odd-cycle contribution $\bar{n}_{\ell}(z) \phi_{\ell}(z)$ scales as $\ell^{-2}$ for large $\ell$, due to the presence of the cut-off, the odd-cycle part of the sum has a correction that scales as $N^{-\frac{1}{2}}$, generating an overall finite-size correction in the AOC that scales as $N^{-\frac{3}{2}}$.

2.3.2. Even-cycle contribution. The contribution of even cycles is found to be small (but in general different from zero) and negative (see figures 2(b) and 3(b) for the values obtained for $z=3$ ). For $z=4$, using a cavity we find $\phi_{4}(4)=-3.56(4) \cdot 10^{-4}$, compared with the numerical estimation $\phi_{4}(4)=-3.6(4) \cdot 10^{-4}$, whereas the value $\phi_{\ell}(4)$ for $\ell \geqslant 6$ is out of the reach of the precision of our numerics. Similarly, the value of $\ell^{-1} \phi_{\ell}(z)$ for $z>4$ has been found to be smaller than $10^{-5}$ in magnitude, and no significant estimation for it has been obtained, neither by using a cavity nor by using the extrapolation 
Random-link matching problems on random regular graphs

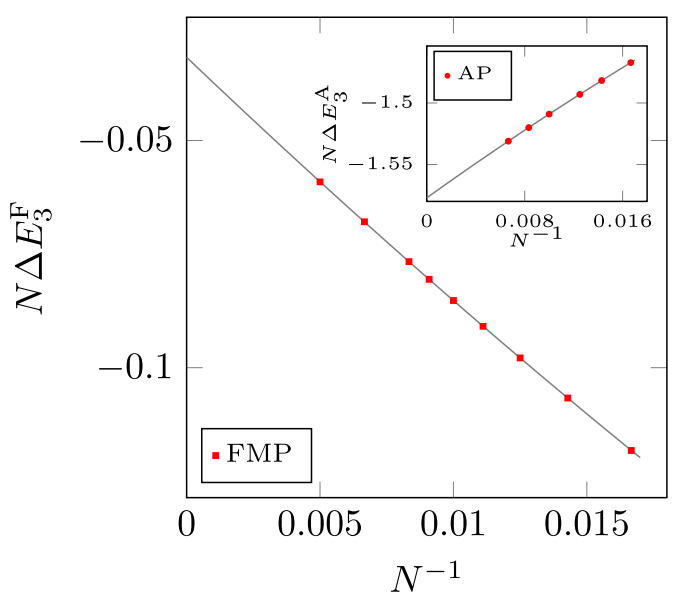

(a)

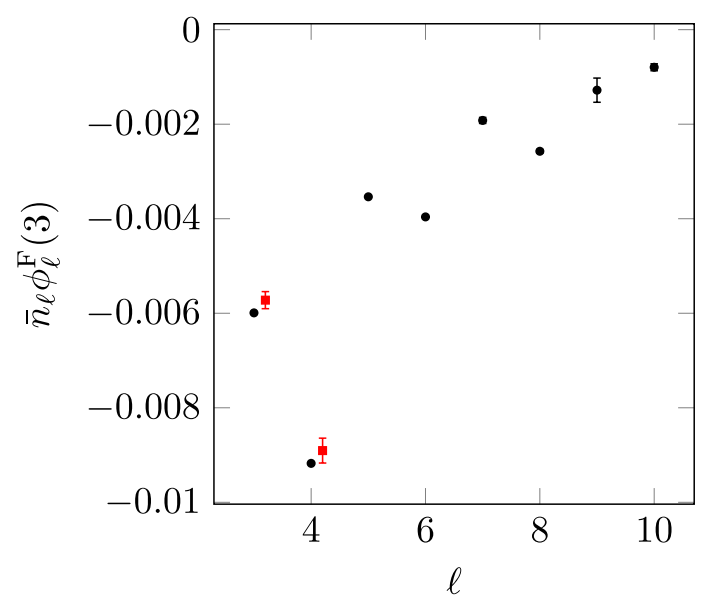

(b)

Figure 3. Cavity and numerical results for the random-link FMP on random regular graphs with $z=3$. (a) Scaling of the finite-size correction for $z=3$ for the $\mathrm{AP}$ and the FMP. See also table 1. On the $y$-axis we have represented for the FMP $N \Delta E_{3}^{\mathrm{F}}:=N\left(E_{3}^{\mathrm{F}}(N)-E_{3}^{\mathrm{F}}\right)$, and similarly for the $\mathrm{AP}, N \Delta E_{3}^{\mathrm{A}}:=N\left(E_{3}^{\mathrm{A}}(N)-E_{3}^{\mathrm{A}}\right)$, where $E_{3}^{\mathrm{A}}=\lim _{N \rightarrow+\infty} E_{3}^{\mathrm{A}}(N)$ is the asymptotic AOC of the AP. The continuous lines are quadratic fits in $\frac{1}{N}$. (b) Value of $\bar{n}_{\ell}(3) \phi_{\ell}(3)$ for the FMP obtained by using a cavity (black circles) and numerically solving the problem (red squares, slightly shifted in the $x$ direction for the sake of clarity). The values for even $\ell$ coincide with the corresponding contribution in the MP.

in equation (41). We recall here that, in the fully connected case, the sum in equation (7) runs over odd contributions only. If the guessed correspondence is true, we expect $\phi_{\ell}(z) \rightarrow 0$ as $z \rightarrow \infty$, with this contribution being small but finite (and negative) at finite $z$. We will further comment on the even-cycle contribution by discussing the random FMP below.

2.3.3. Wrapping up. We have therefore verified (term by term) that cycle contributions can be actually computed using a cavity, and moreover we have given evidences that these cycle contributions asymptotically converge to the terms in equation (7). We now have to sum up all these contributions to get an estimation of $E_{z}^{(1)}$. Let us first consider the odd-cycle contribution for $z=3$. We have good cavity results for cycles up to $\ell=15$,

$$
\sum_{k=1}^{7} \bar{n}_{2 k+1}(3) \phi_{2 k+1}(3)=0.864(2) .
$$

To estimate the contribution of the remaining odd cycles, we extract the asymptotic behavior of the remaining loops using a quartic polynomial in $\frac{1}{\ell}$, obtaining

$$
\sum_{k=8}^{\infty} \bar{n}_{2 k+1}(3) \phi_{2 k+1}(3) \simeq 0.2017(8) .
$$


The total contribution of odd cycles is therefore

$$
\sum_{k=1}^{\infty} \bar{n}_{2 k+1}(3) \phi_{2 k+1}(3) \simeq 1.065(2) .
$$

A similar estimation can be carried out, in principle, for the even-cycle contribution. Unfortunately, the numerics are very noisy, with $\phi_{2 k}(3)$ being very small for $k>4$. Restricting ourselves to the contributions that we have, we obtain

$$
\sum_{k=1}^{4} \bar{n}_{2 k+2}(3) \phi_{2 k+2}(3) \simeq-0.0165(1) .
$$

Summing the two obtained estimations we have

$$
\sum_{k=1}^{\infty} \bar{n}_{2 k+1}(3) \phi_{2 k+1}(3)+\sum_{k=1}^{4} \bar{n}_{2 k+2}(3) \phi_{2 k+2}(3) \simeq 1.048(2),
$$

which is compatible with the direct evaluation $E_{3}^{(1)}=1.045(3)$ obtained via a fit (see table 1). The excellent agreement suggests that the contribution of higher even cycles, and of a possible additive constant $v_{3}$ not depending on the cycles, is negligible at our level of precision. The consistency of equation (24) can be cross-checked by comparing our results with the one corresponding to a variation of the MP that we will discuss below.

\subsection{A note on the assignment problem}

Before studying the FMP, let us make a comment on the so-called assignment problem. By restricting $\mathbb{G}_{\mathrm{RRG}}(N, z)$ to all and only its instances without odd loops, the MP takes the name of the random AP on a Bethe lattice. The AP has the same leading-order behavior of the MP but, due to the bipartite nature of the graph, the contribution of odd cycles is absent in the finite-size corrections by construction, whereas the even-cycle contributions coincide with the ones evaluated for the MP. However, the 'non-topological' additive constant $v_{z}$ that appears in the finite-size corrections is expected to be different from zero. Indeed, the bipartition of the graph induces a gauge invariance in the cavity equation (particularly, an invariance under a shift of the cavity fields) that produces a nontrivial $\frac{1}{N}$ finite-size correction $[15,16]$. Solving the AP on the Bethe lattice, we find no evidence of an $N^{-\frac{3}{2}}$ anomalous correction (as expected if we interpret it as a correction to the odd-cycle contribution in the MP) (see figure 3(a)): the scaling of the AOC with $N$ is therefore exactly of the same type appearing in the fully connected case [30-32]. Because of the arguments given above, the absence of an $N^{-\frac{3}{2}}$ anomalous scaling contribution suggests that, for even $\ell, \bar{n}_{\ell} \phi_{\ell} \sim \ell^{-\alpha}$, with $\alpha \geqslant 3$, or faster, a fact that nevertheless needs a more accurate numerical investigation. Further information about the AOC in the AP can be found in table 1. 


\section{The fractional matching problem}

The results presented in [17] show that the finite-size corrections in the MP can be better understood by relaxing the assumption $m_{e} \in\{0,1\}$ and studying two variants of the problem, namely the random FMP and the random 'loopy' FMP (LMP). In particular, it turns out that the contribution of the sum in equation (7) disappears when switching from the MP to the FMP [42], suggesting that the finite-size corrections are indeed related to the suppression of cycles in the solution of the MP. We follow this hint for the analysis on sparse topologies.

In the so-called random FMP the occupancy variables $m_{e}$ are allowed to take any value in $[0,1]$. As in the MP, given a graph $G=(\mathcal{V} ; \mathcal{E})$, the cost to be minimized is

$$
E_{G}^{\mathrm{F}}[M]:=\frac{1}{|M|} \sum_{e \in \mathcal{E}} w_{e} m_{e},
$$

but this time the constraints read as

$$
m_{e} \in[0,1] \quad \forall e \in \mathcal{E}, \quad \sum_{e \rightarrow v} m_{e}=1 \quad \forall v \in \mathcal{V} .
$$

In the equations above we have defined $|M|:=\sum_{e \in \mathcal{E}} m_{e}=\frac{N}{2}$. As in the MP, we will study the FMP on a random regular graph ensemble $\mathbb{G}_{\mathrm{RRG}}(N, z)$, with link weights drawn according to the distribution of equation (10). The AOC is then defined as

$$
E_{z}^{\mathrm{F}}(N):=\mathbb{E}\left[\min _{M} E_{G}^{\mathrm{F}}[M]\right]
$$

By construction, the AOC $E_{z}^{\mathrm{F}}(N)$ of the FMP on a Bethe lattice of coordination $z$ satisfies the inequality $E_{z}^{\mathrm{F}}(N) \leqslant E_{z}(N)$.

It can be proved that, in the optimal solution of a given instance of the FMP, $m_{e} \in\left\{0, \frac{1}{2}, 1\right\}$ [42]. This result implies that, along with isolated edges, cycles and infinite chains might appear in the optimal matching. However, even-length cycles, although possible in principle, cannot be in the optimal solution: given a cycle $\mathcal{C}$ of even length $\ell$, one of the alternating solutions on it is always cheaper than $\frac{1}{2} \sum_{e \in \mathcal{C}} w_{e}$. Using the same argument, it is easily seen that infinite chains of edges with $m_{e}=\frac{1}{2}$ cannot be in the optimal matching as well. It follows that, since on trees the space of feasible solutions of the MP and the FMP is the same, the analysis of section 2.1 can be repeated here without any modification, and the leading cost is expected to be identical because of this, i.e. $E_{z}^{\mathrm{F}}:=\lim _{N} E_{z}^{\mathrm{F}}(N)=\lim _{N} E_{z}(N)=E_{z}$. This is indeed the case: this fact also implies that, for large $z, E_{z}^{\mathrm{F}}$ behaves as in equation (21), with no $\frac{1}{z}$ corrections. A direct study of the scaling of $E_{z}^{\mathrm{F}}(N)$ with $N$ shows that the MP and the FMP are instead quite different with respect to the scaling of their finite-size corrections: the $N^{-1}$ correction coefficient in the FMP is negative, and there is no $N^{-\frac{3}{2}}$ anomalous contribution (see table 1 and figure 3(a)).

Finite-size corrections are affected by the larger space of feasible solutions in the FMP. We expect the same structure given in equation (24) for the MP. Each cycle contribution, however, must be evaluated by taking into account that an additional solution $m_{e}=\frac{1}{2}$ for each edge $e$ in the cycle is possible, e.g. 


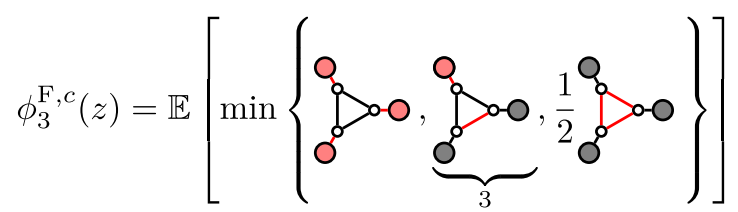

The presence of an additional possible solution does not modify the even-cycle contributions because the 'fractional solution' will never be the solution of minimum cost, but it affects the odd-cycle ones, which, because of the $E_{z}^{\mathrm{F}}(N) \leqslant E_{z}(N)$ inequality, we expect to be smaller than the ones appearing in the MP. This has been verified by using the cavity method and computing directly the cycle contributions (see table 2 and figure 3(b)): in the FMP all cycle contributions are negative, confirming that switching from the FMP to the MP strongly affects the finite-size corrections. The even-cycle contribution of the FMP is identical to the ones appearing in the MP; similarly, cycleindependent correction $v_{z}$, if present, is expected to be the same, because the only modification between the two problems is related to cycles.

We carry out the same analysis performed for the MP for $z=3$, obtaining, for the odd-cycle contribution,

$$
\sum_{k=1}^{\infty} \bar{n}_{2 k+1}(z) \phi_{2 k+1}^{\mathrm{F}}(z) \simeq-0.0153(5) .
$$

Adding the even-cycle terms in equation (45), we have

$$
\sum_{k=1}^{\infty} \bar{n}_{2 k+1}(3) \phi_{2 k+1}^{\mathrm{F}}(3)+\sum_{k=1}^{4} \bar{n}_{2 k+2}(3) \phi_{2 k+2}(3) \simeq-0.0318(6),
$$

which is perfectly compatible with the direct numerical estimation $E_{z}^{\mathrm{F},(1)}=-0.03169(6)$.

Assuming $v_{3}$ to be the same in the FMP and in the MP (possibly zero), we can however perform a consistency check that allows us to recover the $\frac{1}{N}$ correction of the MP from the odd-cycle contributions of the MP and the total $\frac{1}{N}$ correction of the FMP. Observe indeed that the quantity

$$
E_{3}^{(1)}-E_{3}^{\mathrm{F},(1)}=1.077(3),
$$

evaluated using the fit results, should depend on the odd-cycle contributions only, i.e. it should be equal to

$$
\sum_{k=1}^{\infty} \bar{n}_{2 k+1}(3) \phi_{2 k+1}(3)-\sum_{k=1}^{\infty} \bar{n}_{2 k+1}(3) \phi_{2 k+1}^{\mathrm{F}}(3)=1.080(2),
$$

a number obtained using the cavity results. The two values are in perfect agreement.

\section{The ‘loopy’ FMP}

The analysis of the finite-size corrections for the fully connected MP in [17] shows that $\frac{1}{N}$ corrections disappear entirely in the fully connected MP with an exponential weight 
distribution if we allow cycles (as in the FMP) and 'self-matched vertices', i.e. if every vertex can be removed from the graph by paying a price. One might be tempted to perform therefore the same study in the sparse case to get insights on the MP and the FMP, but such a variation can be thought of as a modification of the topology of the graph-due to the addition of $N$ weighted self-loops-that affects not only the finitesize corrections but also the leading order in $N$. The problem is however interesting by itself and, moreover, we will show that some insight on the fully connected problem can be obtained anyway.

To be more precise, in this variant of the FMP, sometimes called random 'loopy' fractional matching [17], an additional non-negative weight $w_{v}$ is associated with each vertex $v \in \mathcal{V}$ of the graph. Each $w_{v}$ is a random variable drawn independently from all other weights with the same probability distribution $\varrho(w)$. An additional 'occupancy variable' $m_{v}$ is associated with each vertex. Given a weighted graph $G=(\mathcal{V} ; \mathcal{E})$, the target is to search for the set $M=\left\{m_{e}\right\}_{e \in \mathcal{E}} \cup\left\{m_{v}\right\}_{v \in \mathcal{V}}$ satisfying the constraints

$$
m_{e} \in[0,1] \quad \forall e \in \mathcal{E}, \quad m_{v} \in[0,1] \quad \forall v \in \mathcal{V}, \quad \sum_{e \rightarrow v} m_{e}+m_{v}=1 \quad \forall v \in \mathcal{V}
$$

that maximizes

$$
|M|=\sum_{e} m_{e}+\frac{1}{2} \sum_{v} m_{v}
$$

and minimizes

$$
E_{G}^{\mathrm{L}}[M]:=\frac{1}{|M|}\left(\sum_{e \in \mathcal{E}} w_{e} m_{e}+\sum_{v \in \mathcal{V}} w_{v} m_{v}\right) .
$$

We will write for the AOC

$$
E_{G}^{\mathrm{L}}(N):=\mathbb{E}\left[\min _{M} E_{G}^{\mathrm{L}}[M]\right] .
$$

Obviously $E_{G}^{\mathrm{L}}(N) \leqslant E_{G}^{\mathrm{F}}(N) \leqslant E_{G}(N)$. In other words, in this problem each vertex can be (partially or totally) matched to itself and removed from the graph, paying a cost $m_{e} w_{v}$. Note that in this case a matching with $|M|=\frac{N}{2}$ always exists. Once again, we are interested in the AOC of the problem in the ensemble of weighted random regular graphs of coordination $z$ and link weights drawn according to the distribution in equation (10).

\subsection{The asymptotic cost}

Similarly to the FMP case, it can be proved that in the optimal configuration $m_{e} \in\left\{0, \frac{1}{2}, 1\right\} \forall e$, and $m_{v} \in\{0,1\} \forall v$ [42], a fact that strongly simplifies the analysis. In addition to this, we can neglect the possibility $m_{e}=\frac{1}{2}$ at the leading order, because of the same arguments given for the FMP for infinite half-occupied chains. Unlike all previously studied problems, the presence of the additional on-vertex degrees of freedom 
modifies the equation and the AOC at the leading level, i.e. on the tree. In particular, we have two types of marginals on the factor graph, the first $\mu$ going from a vertex to a regular edge, and the second one $\hat{\mu}$ going from a vertex $i$ to its self-loop $e_{i}$, occupied by a new variable node

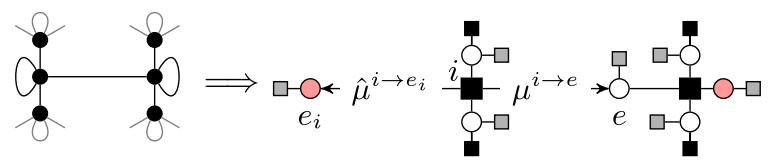

Let us now parametrize $\mu$ and $\hat{\mu}$ in such a way that, at finite temperature,

$$
h^{i \rightarrow e}=-\frac{1}{\beta} \ln \frac{\mu^{i \rightarrow e}(0)}{\mu^{i \rightarrow e}(1)}, \quad s_{i}=-\frac{1}{\beta} \ln \frac{\hat{\mu}^{i \rightarrow e_{i}}(0)}{\hat{\mu}^{i \rightarrow e_{i}}(1)} .
$$

For $\beta \rightarrow+\infty$ we have

$$
\begin{aligned}
& s \stackrel{\mathrm{d}}{=} \min _{1 \leqslant e \leqslant z}\left(w_{e}-h_{e}\right) \\
& h \stackrel{\mathrm{d}}{=} \min \left\{\min _{1 \leqslant e \leqslant z-1}\left(w_{e}-h_{e}\right), w_{v}\right\} .
\end{aligned}
$$

For $w_{v} \rightarrow+\infty$ the cavity equations for the MP/FMP are recovered. If we denote by $p_{z}(h)$ the distribution of $h$ and by $\hat{p}_{z}(s)$ the distribution of $s$, the AOC is given by

$$
\begin{aligned}
E_{z}^{\mathrm{L}}=z \int \mathrm{d} w w \varrho_{z}(w) \iint \mathrm{d} h \mathrm{~d} h^{\prime} \theta\left(h+h^{\prime}-w\right) p_{z} & (h) p_{z}\left(h^{\prime}\right) \\
& +2 \int \mathrm{d} w w \varrho_{z}(w) \int \mathrm{d} s \hat{p}_{z}(s) \theta(s-w) .
\end{aligned}
$$

The asymptotic AOC $E_{z}^{\mathrm{L}} \neq E_{z}$ is found to satisfy a different scaling relation in $z$ with respect to the $\mathrm{MP}$ and the FMP. In particular, we find

$$
E_{z}^{\mathrm{L}}=\frac{\zeta(2)}{2}-\frac{\zeta(2)}{2 z}+o\left(\frac{1}{z}\right)
$$

i.e. a negative linear term in $\frac{1}{z}$ is present. Equation (57) is proven in the appendix. We numerically verify the cavity predictions for $3 \leqslant z \leqslant 15$ (see figure 4 (a)). The $\frac{1}{z}$ correction at the leading order is due to the presence of self-loops, which are occupied with finite probability. The second integral in equation (56), in particular, corresponds to the average self-loop cost $\phi_{1}^{\mathrm{L}}(z)$. Observing that $s$ and $h$ are identically distributed for $z \rightarrow+\infty$, it can be evaluated exactly for $z \gg 1$ as

$$
\phi_{1}^{\mathrm{L}}(z):=\int \mathrm{d} w w \varrho_{z}(w) \int \mathrm{d} s \hat{p}_{z}(s) \theta(s-w)=\frac{\zeta(2)}{4 z}+o\left(\frac{1}{z}\right)
$$

[see figure 4(b)]. The probability that a vertex is 'self-matched', on the other hand, decreases as (see figure 4(b))

$$
p_{s}(z)=\int \mathrm{d} w \varrho_{z}(w) \int \mathrm{d} s \hat{p}_{z}(s) \theta(s-w)=\frac{\ln 2}{z}+o\left(\frac{1}{z}\right) .
$$




\subsection{Finite-size corrections}

Finite-size corrections are also expected to be affected by the larger space of feasible solutions. The LMP has no anomalous corrections (see figure 4(a)), like the FMP. However, both even- and odd-cycle contributions are different with respect to the corresponding ones in the MP and the FMP (see table 2). As in the previous cases, each cycle contribution must be evaluated by taking into account all allowed configurations in the FMP, plus the possibility that the vertices are self-matched. Pictorially,

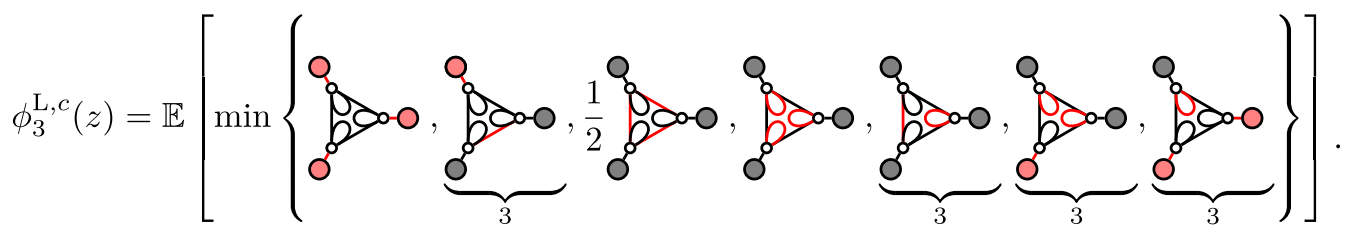

As in the FMP, $\bar{n}_{\ell}(z) \phi_{\ell}^{\mathrm{L}}(z)$ decays to zero very fast both in $\ell$ and in $z$, making the numerical evaluation for $z \geqslant 4$ and $\ell \geqslant 4$ very difficult. In figure 4(d) we present our cavity results for $\bar{n}_{3} \phi_{3}^{\mathrm{L}}(z)$, which appears to go to zero exponentially fast in $z$. For $z=3$, the computation is less cumbersome: the cavity results for the corrections in the $z=3$ case are given in table 2 , and in figure 4(c), where they are compared with the numerical estimations obtained for $\ell=3$ and $\ell=4$. As in the MP, $\phi_{\ell}^{\mathrm{L}}(3)$ is found to be positive for odd cycles and negative for even cycles. The sum of the first contributions is

$$
\sum_{\ell=3}^{9} \bar{n}_{\ell}(3) \phi_{\ell}^{\mathrm{L}}(3)=0.0166(2),
$$

which is close to, but not compatible with, the result obtained from a fit, given in table $1, E_{z}^{\mathrm{L},(1)}=0.0154(2)$, due to the fact that all contributions for $\ell>9$ have been neglected. The quantity $\left|\bar{n}_{\ell} \phi_{\ell}^{\mathrm{L}}(3)\right|$ is found to go to zero exponentially fast (see figure 4(c)). Extrapolating through the fit parameters, we estimate

$$
\sum_{\ell=3}^{\infty} \bar{n}_{\ell}(3) \phi_{\ell}^{\mathrm{L}}(3) \simeq 0.0153(5),
$$

which is compatible with the fit result. As in the MP and the FMP, no additional constant is found within the precision of our calculations.

\section{The fully connected limit}

As anticipated, the obtained results shed some light on the nature of the finite-size corrections in the fully connected model. With the exception of the odd-cycle contribution in the MP, we have observed that all cycle corrections go to zero as $z \rightarrow+\infty$ in all considered models, at least within our numerical precision. Our results suggest that, for large $N$

$$
\lim _{z \rightarrow N-1} E_{z}(N)=\frac{\zeta(2)}{2}+\frac{1}{N} \sum_{k=1}^{\infty} \lim _{z \rightarrow \infty} \bar{n}_{k}(z) \phi_{k}(z)+o\left(\frac{1}{N}\right)=\frac{\zeta(2)}{2}+\frac{2}{N} \sum_{k=1}^{\infty} \frac{I_{2 k+1}}{2 k+1}+o\left(\frac{1}{N}\right) .
$$


Random-link matching problems on random regular graphs

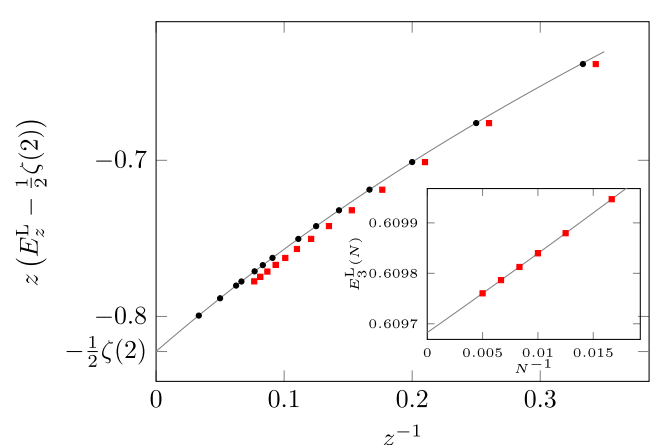

(a)

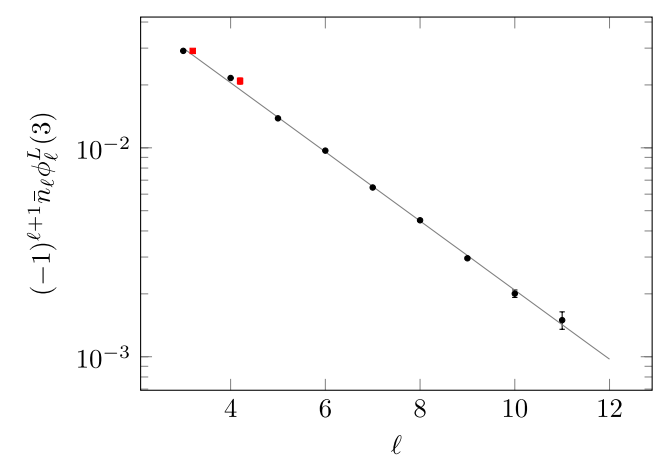

(c)

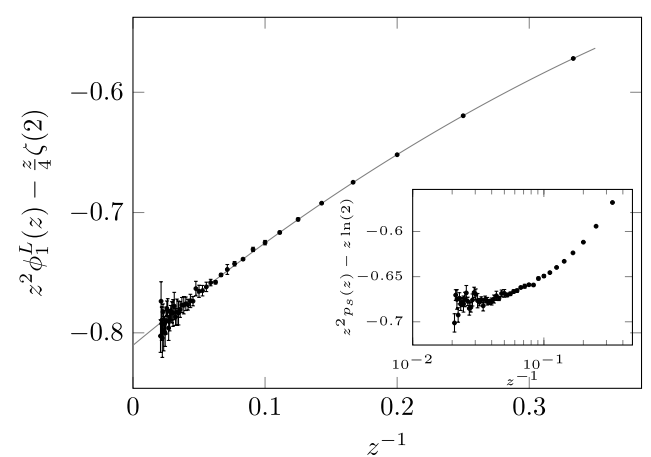

(b)

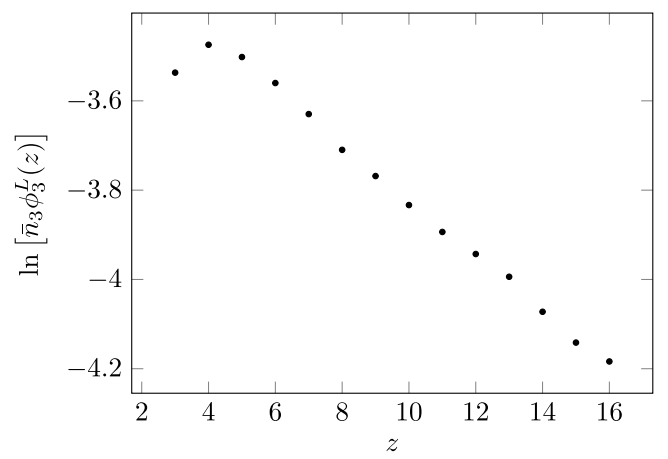

(d)

Figure 4. Cavity and numerical results for the random LMP. (a) AOC for the LMP as a function of $z$. The asymptotic correction to the mean field value scales as $z^{-1}$. Numerical simulations (red squares, shifted by $10^{-2}$ in the $x$ direction for the sake of clarity) are in perfect agreement with the cavity prediction (black circles). A cubic fit in $\frac{1}{z}$ is also represented. In the inset, the cost density of the LMP for $z=3$ is shown as a function of $\frac{1}{N}$. The continuous line is a quadratic fit in $\frac{1}{N}$. (b) Cavity estimation of the average cost $\phi_{1}^{\mathrm{L}}(z)$ of a self-loop as a function of $z$ in the LMP. In the inset, the occupation probability $p_{s}(z)$ of the self-loop is shown as a function of $z$. Note that the asymptotic value is given by $\lim _{z \rightarrow \infty} z p_{s}(z)=\ln 2$ and higher-order corrections scale as $\frac{1}{z^{2}}$. (c) Value of $\bar{n}_{\ell}(3) \phi_{\ell}(3)$ for the LMP. Numerical results (red squares, shifted in the $x$ direction for the sake of clarity) are compared with the cavity predictions (black circles). The observed decay is exponential. The gray curve corresponds to an exponential fit of the type $f(\ell)=a \mathrm{e}^{-b \ell}$, with $a=0.094(4)$ and $b=0.381(5)$. (d) Loop contribution for $\ell=3$ as a function of $z$ in the LMP obtained using the cavity method.

Similarly, for the FMP, no $\frac{1}{N}$ finite-size correction is expected for $z \rightarrow N-1$.

These predictions, based on the assumption that the sparse-dense correspondence holds also at the level of finite-size corrections, are equal to the exact results obtained on the complete graph $K_{N}$ given in [17], up to an additional correction, which is the same in all cases and equal to $\frac{\zeta(2)}{2 N}$, as can be seen from equation (7) with reference to the MP. This apparent disagreement is however only due to a different choice of the weight distribution density. To better understand the subtlety behind this apparent discrepancy, let us restrict ourselves to the MP. In [17], the authors consider a cost function $\hat{E}_{K_{N}}[M]$ that is slightly different from the one in equation (3), i.e. 


$$
\hat{E}_{K_{N}}[M]=\sum_{e} m_{e} \hat{w}_{e}=\frac{2}{N-1} \sum_{e} m_{e} w_{e}=\frac{N}{N-1} E_{K_{N}}[M],
$$

where the quantities $\hat{w}_{e}$ are i.i.d. random variables extracted from $\hat{\varrho}(\hat{w})=\mathrm{e}^{-\hat{w}}$, and we have performed a change of variables $w=\frac{N-1}{2} \hat{w}$, so that $\hat{w}$ is extracted from $\varrho_{N-1}(w)$, as should be in our convention. By consequence, the cost in [17] is such that

$$
\hat{E}_{K_{N}}=\frac{N}{N-1} E_{K_{N}}=E_{K_{N}}+\frac{E_{K_{N}}}{N}+o\left(\frac{1}{N}\right) .
$$

For the same reason, this correction appears both in the FMP and in the LMP.

Aside from this contribution, which only appears because of different conventions in the definition of the cost, the fully connected case is recovered by taking $z \rightarrow N-1$. For finite $z$, the leading term $E_{z}$ in the MP and the FMP has no $\frac{1}{z}$ correction, so taking $z \rightarrow N-1$ does not give additional $\frac{1}{N}$ terms, and the fully connected limit is recovered. In the LMP, instead, a $\frac{1}{z}$ correction is present (see equation (57)). Taking $z \rightarrow N-1$ and observing that the cycle contribution goes to zero for $z=N-1 \rightarrow+\infty$, we have

$$
\lim _{z \rightarrow N-1} E_{z}^{\mathrm{L}}(N)=\frac{\zeta(2)}{2}-\frac{\zeta(2)}{2 N}+o\left(\frac{1}{N}\right)
$$

In particular, adopting the convention of [17], the additional contribution exactly cancels the $\frac{1}{N}$ correction above, giving no $\frac{1}{N}$ terms.

\section{Conclusions}

In the present work we have analyzed the random-link matching problem on random regular graphs of coordination $z$, along with two variants of the problem, namely the fractional matching problem and the 'loopy' fractional matching problem. In all cases, the AOC has been computed using the cavity method, and compared with numerical results obtained by solving a large number of instances of the corresponding problem. In the spirit of previous finite-size analyses of disordered systems on sparse topologies, we have also evaluated the finite-size corrections, assuming that they can be decomposed in contributions of single topological structures (cycles herein) appearing in the graph with density $O\left(\frac{1}{N}\right)$. Due to the fact that each cycle of length $\ell$ can be thought of as embedded in an infinite tree, its average contribution to the cost $\phi_{\ell}(z)$ can be evaluated, once again, by means of the cavity method, and must then be reweighted by its multiplicity $\bar{n}_{\ell}(z)$. The quantities $\phi_{\ell}(z)$ go to zero as $z^{-\ell}$, or faster, for large values of $z$ and $\ell$ : the analysis has been carried out therefore mostly for $z=3$. Our results can be summarized as follows.

In the random-link matching problem, odd cycles and even cycles are found to contribute very differently to the finite-size corrections. Odd-cycle terms $\bar{n}_{\ell} \phi_{\ell}$ are positive and scale as $\ell^{-2}$ for large $\ell$, implying the presence of an additional anomalous $N^{-\frac{3}{2}}$ correction, which is indeed found in our numerical results. Even-cycle contributions, on the other hand, are found to be negative and smaller in modulus, with a faster decay 
in $\ell$ and infinitesimal size for large $z$ (the very fast decay in $z$ and the small modulus did not allow us to extract the decaying properly). We have also found strong evidence that, for $\ell=3$ and $\ell=5, \bar{n}_{\ell} \phi_{\ell}$ converges to a corresponding 'odd-cycle-like' contribution appearing in the finite-size corrections of the fully connected problem. The correspondence of the fully connected finite-size expansion to actual cycles in the graph has been suggested in [16, 17, 29], but not directly verified: our results support this claim and the consequent argument given in [29] for the anomalous correction appearing on the complete graph. Remarkably, the cavity prediction for each cycle contribution is in agreement with the numerical estimation obtained by solving the problem directly, whenever such an estimation is practicable. The sum of all cycle contributions evaluated using the cavity method is fully compatible with our numerical results for the $\frac{1}{N}$ correction in the random-link matching problem. In this way, our ansatz for the finitesize correction has been verified both globally and term by term.

As an additional cross-check, we have also considered the so-called fractional matching problem on random regular graphs. In this version of the matching problem, an optimal configuration can be made of both odd cycles and dimers. We have found that fractional matching and the regular type are asymptotically equivalent for any value of the coordination $z$, but they differ in finite-size corrections. As expected, only the odd-cycle corrections are found to be different with respect to the standard matching, their scaling with $\ell$ being faster than $\ell^{-3}$ : this implies no $N^{-\frac{3}{2}}$ correction in fractional matching, a fact that has been verified numerically. Once again, within the precision of our cavity results, the finite-size corrections found numerically are compatible with our cycle expansion.

Finally, we have studied, in the same way, the so-called 'loopy' fractional matching problem, which is a variant of the fractional matching problem in which a vertex can be removed by paying a price of 'self-matching'. For finite $z$, vertices are self-matched with finite probability. Both the leading cost and the finite-size correction can be estimated using the cavity method, the latter by means of the usual cycle expansion.

Using the cavity method, we have computed analytically the $O\left(\frac{1}{z}\right)$ corrections to the asymptotic AOC in all the discussed problems. Moreover, with the exception of the AP, we have found no numerical evidence of additional cycle-independent contributions, although they cannot be excluded a priori. Such contributions might in general appear with a different choice of the weight distribution $\varrho$, as happens on a complete graph $[16,17]$.

\section{Acknowledgments}

The authors would like to thank Enzo Marinari, Federico Ricci-Tersenghi and Tommaso Rizzo for useful discussions. We also thank the anonymous referees for the careful reading of the manuscript and valuable suggestions that improved the readability of the paper. The research of GP and GS has been supported by the Simons Foundation (Grant No. 454949, G Parisi).

\section{Appendix. Finite-z corrections}

In this appendix we study equation (20) for large values of $z$. Let us start denoting by 


$$
\Phi_{z}(x):=\int_{x}^{\infty} p_{z}(h) \mathrm{d} h=\Phi(x)+\frac{\Phi^{(1)}(x)}{z}+o\left(\frac{1}{z}\right),
$$

where $\Phi(x)=\lim _{z \rightarrow+\infty} \Phi_{z}(x)$. The equation for $\Phi(x)$ is obtained from equation (20) by taking $z \rightarrow \infty$,

$$
\Phi(x)=\exp \left(-2 \int_{0}^{\infty} \Phi(w-x) \mathrm{d} w\right)
$$

which has the solution [13]

$$
\Phi(x)=\lim _{z \rightarrow+\infty} \Phi_{z}(x)=\frac{1}{1+\mathrm{e}^{2 x}} \Rightarrow p(h)=\frac{2 \mathrm{e}^{2 h}}{\left(1+\mathrm{e}^{2 h}\right)^{2}} .
$$

In order to compute the finite connectivity corrections to $E_{\infty}$, let us expand the cavity field distribution around the limit (A.3):

$$
p_{z}(h)=-\frac{\mathrm{d} \Phi_{z}(h)}{\mathrm{d} h}=p(h)+\frac{p^{(1)}(h)}{z}+o\left(\frac{1}{z}\right)
$$

from which we have

$$
\begin{aligned}
E_{z}=2 \int_{0}^{+\infty} \mathrm{d} w w \iint & \mathrm{d} h_{1} \mathrm{~d} h_{2} \theta\left(h_{1}+h_{2}-w\right) p\left(h_{1}\right) p\left(h_{2}\right) \\
& -\frac{4}{z} \int_{0}^{+\infty} \mathrm{d} w w^{2} \iint \mathrm{d} h_{1} \mathrm{~d} h_{2} \theta\left(h_{1}+h_{2}-w\right) p\left(h_{1}\right) p\left(h_{2}\right) \\
& +\frac{4}{z} \int_{0}^{+\infty} \mathrm{d} w w \iint \mathrm{d} h_{1} \mathrm{~d} h_{2} \theta\left(h_{1}+h_{2}-w\right) p^{(1)}\left(h_{1}\right) p\left(h_{2}\right)+o\left(\frac{1}{z}\right),
\end{aligned}
$$

which, by using the explicit expression of $p(h)$, becomes

$$
E_{z}=\frac{\zeta(2)}{2}-\frac{2 \zeta(3)}{z}+\frac{2}{z} \int \mathrm{d} h \Phi^{(1)}(h) \ln \left(1+\mathrm{e}^{2 h}\right)+o\left(\frac{1}{z}\right) .
$$

To evaluate $\Phi^{(1)}(x)=\int_{x}^{+\infty} p^{(1)}(h) \mathrm{d} h$ we start from its definition

$$
\Phi^{(1)}(x)=-\lim _{z \rightarrow+\infty} z^{2} \frac{\mathrm{d} \Phi_{z}(x)}{\mathrm{d} z}=-\lim _{z \rightarrow+\infty} z^{2} \frac{\mathrm{d}}{\mathrm{d} z}\left(1-\mathbb{E}\left[\Phi_{z}(w-x)\right]\right)^{z-1} .
$$

Observe now that

$$
\begin{aligned}
\mathbb{E}\left[\Phi_{z}(w-x)\right]= & \frac{2}{z} \int_{0}^{\infty} \mathrm{e}^{-\frac{2 w}{z}} \Phi_{z}(w-x) \mathrm{d} w \\
& ==\frac{\ln \left(1+\mathrm{e}^{2 x}\right)}{z}+\frac{1}{z^{2}}\left(2 \int_{0}^{\infty} \Phi^{(1)}(w-x) \mathrm{d} w+\operatorname{Li}_{2}\left(-\mathrm{e}^{2 x}\right)\right)+o\left(\frac{1}{z^{2}}\right),
\end{aligned}
$$

where we have introduced a polylogarithm of order $s$,

$$
\operatorname{Li}_{s}(z):=\sum_{p=1}^{\infty} \frac{z^{p}}{p^{s}}, \quad|z|<1
$$


Random-link matching problems on random regular graphs

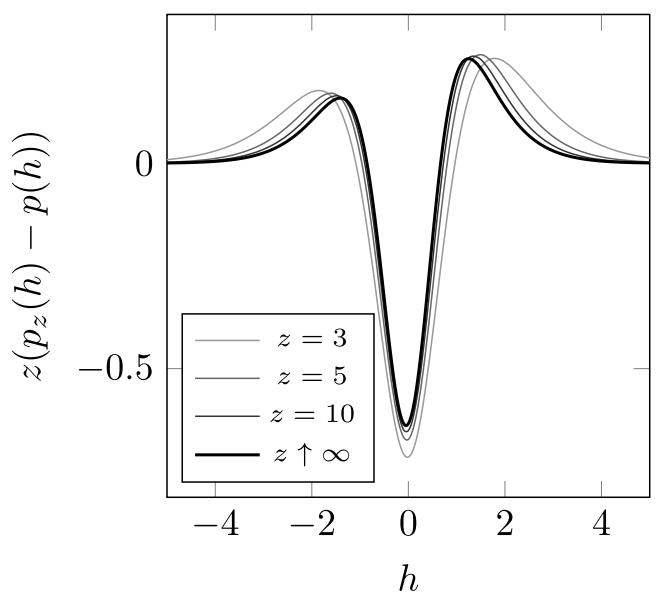

(a)

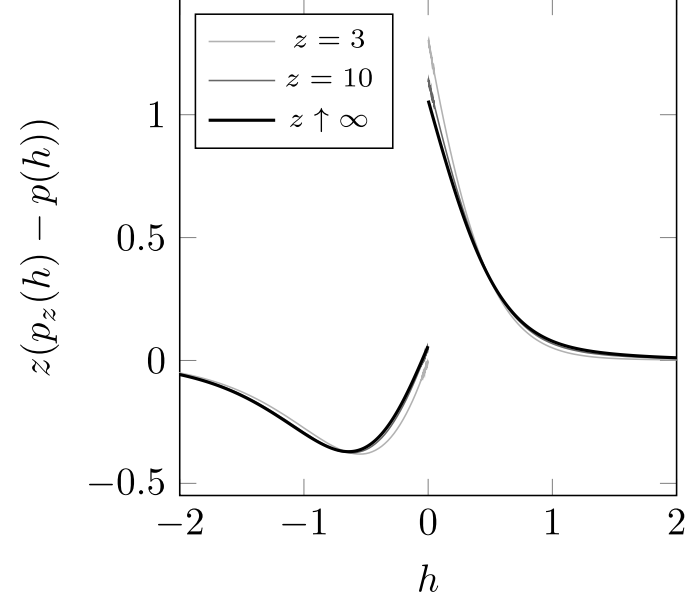

(b)

Figure A1. Plots of the $\frac{1}{z}$ corrections to the cavity field distribution in the analyzed random matching problems. The finite- $z$ results have been obtained using a population dynamics algorithm. (a) Plot of the $\frac{1}{z}$ correction to the $z \rightarrow+\infty$ cavity field probability distribution for the MP and the FMP. (b) Plot of the $\frac{1}{z}$ correction to the $z \rightarrow+\infty$ cavity field probability distribution for the LMP.

defined over $\mathbb{C}$ by analytical continuation. We can write a Volterra integral equation

$$
\Psi(x)+2 \int_{-x}^{\infty} \Phi(t) \Psi(t) \mathrm{d} t=A(x),
$$

for the auxiliary function

$$
\Psi(x):=\frac{\Phi^{(1)}(x)}{\Phi(x)}
$$

where we have introduced

$$
A(x):=\ln \left(1+\mathrm{e}^{2 x}\right)-\frac{\ln ^{2}\left(1+\mathrm{e}^{2 x}\right)}{2}-\mathrm{Li}_{2}\left(-\mathrm{e}^{2 x}\right) .
$$

A differential equation for $\Psi$ is immediately written as

$$
\Psi^{\prime}(x)+2 \Phi(-x) \Psi(-x)=A^{\prime}(x),
$$

or, equivalently, as a system of differential equations for the symmetric and the antisymmetric parts of $\Psi$,

$$
\left\{\begin{array}{l}
\Psi_{\mathrm{s}}^{\prime}(x)=A_{\mathrm{s}}^{\prime}(x)+\Psi_{\mathrm{a}}(x)-\tanh (x) \Psi_{\mathrm{s}}(x), \\
\Psi_{\mathrm{a}}^{\prime}(x)=A_{\mathrm{a}}^{\prime}(x)-\Psi_{\mathrm{s}}(x)+\tanh (x) \Psi_{\mathrm{a}}(x) .
\end{array}\right.
$$

Here we have used the fact that $\Phi(x)+\Phi(-x)=1$ and $\Phi(x)-\Phi(-x)=-\tanh (x)$. Moreover, given a generic function $f(x)$, we have used the notation

$$
f_{\mathrm{s}}(x):=\frac{f(x)+f(-x)}{2}, \quad f_{\mathrm{a}}(x):=\frac{f(x)-f(-x)}{2},
$$


for its symmetric and antisymmetric parts, respectively. After simple manipulations, we obtain

$$
\left\{\begin{array}{l}
\Psi_{\mathrm{a}}^{\prime \prime}(x)=A_{\mathrm{a}}^{\prime \prime}(x)-A_{\mathrm{s}}^{\prime}(x)+\tanh (x) A_{\mathrm{a}}^{\prime}(x), \\
\Psi_{\mathrm{a}}^{\prime}(x)=A_{\mathrm{a}}^{\prime}(x)-\Psi_{\mathrm{s}}(x)+\tanh (x) \Psi_{\mathrm{a}}(x) .
\end{array}\right.
$$

We can solve directly for $\Psi_{\mathrm{a}}$ using the first equation. To fix the two integration constants that appear in the integration, we use the fact that $\lim _{x \rightarrow \pm \infty} \Phi^{(1)}(x)=0$, and the symmetry conditions (which imply $\Psi_{\mathrm{a}}(0)=0$ ). We obtain

$$
\Psi_{\mathrm{a}}(x)=-\frac{\zeta(3)}{2}+x+\int_{-\infty}^{x} \ln \left(1+\mathrm{e}^{-2 t}\right) \ln \left(1+\mathrm{e}^{2 t}\right) \mathrm{d} t .
$$

From $\Psi_{\mathrm{a}}(x)$ we can directly obtain $\Phi^{(1)}(x)$ as

$$
\Phi^{(1)}(x)=\Phi(x)\left[\Psi_{\mathrm{a}}(x)+\Psi_{\mathrm{s}}(x)\right]=\frac{A_{\mathrm{a}}^{\prime}(x)}{1+\mathrm{e}^{2 x}}-\frac{\mathrm{d}}{\mathrm{d} x}\left[\Psi_{\mathrm{a}}(x) \Phi(x)\right] .
$$

This expression gives us $p^{(1)}(h)$ (see figure A1(a)). The explicit expression of $\Psi_{\mathrm{a}}(x)$ is, however, not necessary to calculate the integral in equation (A.6). Indeed, let us first observe that, due to the fact that $\Phi(-x)=\mathrm{e}^{2 x} \Phi(x)$,

$$
\Psi_{\mathrm{a}}(x) \Phi(x)=\frac{\Phi(x)}{2}\left[\frac{\Phi^{(1)}(x)}{\Phi(x)}-\frac{\Phi^{(1)}(-x)}{\Phi(-x)}\right]=\frac{\Phi^{(1)}(x)-\mathrm{e}^{-2 x} \Phi^{(1)}(-x)}{2} .
$$

Inserting equation (A.18) in equation (A.6), the last integral contains in particular the term

$$
\begin{aligned}
-\int_{-\infty}^{\infty} \frac{\mathrm{d}}{\mathrm{d} x}\left[\Psi_{\mathrm{a}}(x) \Phi(x)\right] \ln \left(1+\mathrm{e}^{2 x}\right) \mathrm{d} x & =\int_{-\infty}^{+\infty} \frac{\mathrm{e}^{2 x} \Phi^{(1)}(x)-\Phi^{(1)}(-x)}{1+\mathrm{e}^{2 x}} \mathrm{~d} x \\
& =\int_{-\infty}^{+\infty} \frac{\Phi^{(1)}(x)}{1+\mathrm{e}^{-2 x}} \mathrm{~d} x-\int_{-\infty}^{+\infty} \frac{\Phi^{(1)}(-x)}{1+\mathrm{e}^{2 x}} \mathrm{~d} x=0 .
\end{aligned}
$$

Equation (A.6) reduces to

$$
E_{z}=\frac{\zeta(2)}{2}-\frac{2 \zeta(3)}{z}+\frac{2}{z} \int \mathrm{d} h A_{\mathrm{a}}^{\prime}(h) \frac{\ln \left(1+\mathrm{e}^{2 h}\right)}{1+\mathrm{e}^{2 x}}+o\left(\frac{1}{z}\right) .
$$

Plugging in the equation the explicit expression of $A_{\mathrm{a}}^{\prime}(x)$, we have found that the last integral is equal to $\zeta(3)$. The $\frac{1}{z}$ correction in the MP is therefore equal to zero, as numerically verified.

\section{A.1. Loopy fractional random matching}

The arguments above can be repeated in the case of the LMP. Let

$$
\Phi_{z}(x)=\int_{x}^{\infty} p_{z}(h) \mathrm{d} h, \quad \hat{\Phi}_{z}(x)=\int_{x}^{\infty} \hat{p}_{z}(s) \mathrm{d} s .
$$

Equations (55) imply 


$$
\begin{aligned}
& \Phi_{z}(x)=\left(1-\mathbb{E}\left[\Phi_{z}(w-x)\right]\right)^{z-1} \int_{x}^{\infty} \varrho_{z}(w) \mathrm{d} w=\mathrm{e}^{-\frac{2 x}{z} \theta(x)}\left(1-\mathbb{E}\left[\Phi_{z}(w-x)\right]\right)^{z-1}, \\
& \hat{\Phi}_{z}(x)=\left(1-\mathbb{E}\left[\Phi_{z}(w-x)\right]\right)^{z} .
\end{aligned}
$$

Let us start from the equation for $\Phi_{z}(h)$. It is evident that, for $z \rightarrow+\infty, \Phi_{z}(x) \rightarrow \Phi(x)$ as in the MP. Proceeding as in the standard case (and following the same notation) we obtain the same integral equation (equation (A.13)), but with a different function $A(x)$, namely

$$
A(x)=\ln \left(1+\mathrm{e}^{2 x}\right)-\frac{\ln ^{2}\left(1+\mathrm{e}^{2 x}\right)}{2}-\mathrm{Li}_{2}\left(-\mathrm{e}^{2 x}\right)-2 x \theta(x) .
$$

The next steps follow exactly the MP case. We obtain an expression for the antisymmetric part $\Psi_{\mathrm{a}}(x)$ of $\Psi(x)=\frac{\Phi^{(1)}}{\Phi(x)}$ as follows:

$\Psi_{\mathrm{a}}(x)=\frac{\zeta(2)-2 \zeta(3)}{4}+x^{2} \theta(x)+\frac{1}{2} \mathrm{Li}_{2}\left(-\mathrm{e}^{2 x}\right)+\int_{-\infty}^{x} \ln \left(1+\mathrm{e}^{-2 t}\right) \ln \left(1+\mathrm{e}^{2 t}\right) \mathrm{d} t$

from which the expression of $\Phi^{(1)}$, and then $p^{(1)}(h)$, can be obtained (see figure A1(b)). As before, the $\frac{1}{z}$ correction is obtained from $A_{\mathrm{a}}(x)$ : from equation (56), we have the requirement that an additional $\frac{1}{z}$ correction must be included, due to self-loops and as given in equation (58), obtaining

$$
\begin{aligned}
E_{z}^{\mathrm{L}}=\frac{\zeta(2)}{2}+ & \frac{\zeta(2)}{2 z}-\frac{2 \zeta(3)}{z}+\frac{2}{z} \int \mathrm{d} h A_{\mathrm{a}}^{\prime}(h) \frac{\ln \left(1+\mathrm{e}^{2 h}\right)}{1+\mathrm{e}^{2 x}}+o\left(\frac{1}{z}\right) \\
& =\frac{\zeta(2)}{2}+\frac{\zeta(2)}{2 z}-\frac{2}{z} \int \mathrm{d} h \frac{\ln \left(1+\mathrm{e}^{2 h}\right)}{1+\mathrm{e}^{2 x}}+o\left(\frac{1}{z}\right)=\frac{\zeta(2)}{2}-\frac{\zeta(2)}{2 z}+o\left(\frac{1}{z}\right) .
\end{aligned}
$$

\section{References}

[1] Lovász L and Plummer D 2009 Matching Theory (Providence, RI: AMS Chelsea Publishing Series)

[2] Kasteleyn P W 1967 Graph theory and crystal physics Graph Theory and Theoretical Physics (New York: Academic) pp 43-110

[3] Papadimitriou C and Steiglitz K 1998 Combinatorial Optimization: Algorithms and Complexity (Dover Books on Computer Science Series) (New York: Dover)

[4] Kuhn H W 1955 Nav. Res. Logist. Q. 2 83-97

[5] Edmonds J 1965 Can. J. Math. 17 449-67

[6] Bayati M, Shah D and Sharma M 2008 IEEE Trans. Inf. Theory 54 1241-51

[7] Kirkpatrick S, Gelatt C and Vecchi M 1983 Science 220 671-80

[8] Mézard M, Parisi G and Virasoro M 1987 Spin Glass Theory and Beyond (Lecture Notes in Physics Series) (Singapore: World Scientific)

[9] Mézard M, Parisi G and Zecchina R 2002 Science 297 812-5

[10] Krzakała F, Montanari A, Ricci-Tersenghi F, Semerjian G and Zdeborová L 2007 Proc. Natl Acad. Sci. $10410318-23$

[11] Mézard M and Montanari A 2009 Information, Physics, and Computation (Oxford Graduate Texts) (Oxford: Oxford University Press)

[12] Orland H 1985 J. Phys. Lett. 46 763-70

[13] Mézard M and Parisi G 1985 J. Phys. Lett. 46 771-8

[14] Mézard M and Parisi G 1986 Europhys. Lett. 2 913-8

[15] Mézard M and Parisi G 1987 J. Phys. 48 1451-9 
[16] Parisi G and Ratiéville M 2002 Eur. Phys. J. B 29 457-68

[17] Lucibello C, Malatesta E M, Parisi G and Sicuro G 2018 J. Stat. Mech. 053301

[18] Malatesta E M, Parisi G and Sicuro G 2019 Phys. Rev. E 100032102

[19] Caracciolo S, Lucibello C, Parisi G and Sicuro G 2014 Phys. Rev. E 90012118

[20] Caracciolo S and Sicuro G 2015 Phys. Rev. E 91062125

[21] Caracciolo S and Sicuro G 2015 Phys. Rev. Lett. 115230601

[22] Zhou H and Ou-Yang Z C 2003 (arXiv:0309348)

[23] Zdeborová L and Mézard M 2006 J. Stat. Mech. P05003

[24] Bordenave C, Lelarge M and Salez J 2013 Probab. Theory Relat. Fields 157 183-208

[25] Montanari A and Rizzo T 2005 J. Stat. Mech. P10011

[26] Ferrari U, Lucibello C, Morone F, Parisi G, Ricci-Tersenghi F and Rizzo T 2013 Phys. Rev. B 88184201

[27] Lucibello C, Morone F, Parisi G, Ricci-Tersenghi F and Rizzo T 2014 Phys. Rev. E 90012146

[28] Coja-Oghlan A, Efthymiou C, Jaafari N, Kang M and Kapetanopoulos T 2018 Commun. Math. Phys. $359603-98$

[29] Lucibello C, Parisi G and Sicuro G 2017 Phys. Rev. E 95012302

[30] Parisi G 1998 (arXiv:9801176)

[31] Linusson S and Wästlund J 2004 Probab. Theory Relat. Fields 128 419-40

[32] Nair C, Prabhakar B and Sharma M 2005 Random Struct. Algorithms 27 413-44

[33] Lucibello C, Morone F and Rizzo T 2014 Phys. Rev. E 90012140

[34] Bollobás B 2001 Random Graphs (Cambridge Studies in Advanced Mathematics) 2nd edn (Cambridge: Cambridge University Press)

[35] McKay B D and Wormald N C 1984 Combinatorica 4 325-38

[36] Wormald N C 1999 Models of random regular graphs Surveys in Combinatorics ed J D Lamb and D A Preece (Cambridge: Cambridge University Press) pp 239-98

[37] Mézard M and Parisi G 2001 Eur. Phys. J. B 20 217-33

[38] Bollobás B and McKay B D 1986 J. Comb. Theory B 41 80-91

[39] Korada S B and Montanari A 2011 IEEE Trans. Inf. Theory 57 2440-50

[40] Dezsö B, Jüttner A and Kovács P 2011 Electron. Notes Theor. Comput. Sci. 264 23-45

[41] Wormald N C 1981 J. Comb. Theory B 31 168-82

[42] Wästlund J 2010 Acta Math. 204 91-150 